\title{
Detection on Structural Sudden Damage Using Continuous Wavelet Transform and Lipschitz Exponent
}

\author{
Bo Chen, ${ }^{1}$ Yuan-pin Kang, ${ }^{1}$ Peng-yun Li, ${ }^{2}$ and Wen-ping Xie ${ }^{2}$ \\ ${ }^{1}$ Key Laboratory of Roadway Bridge and Structural Engineering, Wuhan University of Technology, Mail Box No. 219, \\ No. 122 Luoshi Road, Wuhan 430070, China \\ ${ }^{2}$ Guangdong Power Grid Corporation Co. Ltd., Guangzhou 510080, China \\ Correspondence should be addressed to Bo Chen; cbsteven@163.com
}

Received 3 August 2014; Accepted 12 October 2014

Academic Editor: Ting-Hua Yi

Copyright (C) 2015 Bo Chen et al. This is an open access article distributed under the Creative Commons Attribution License, which permits unrestricted use, distribution, and reproduction in any medium, provided the original work is properly cited.

\begin{abstract}
The degradation of civil engineering structures may lead to a sudden stiffness reduction in a structure and such a sudden damage will cause a discontinuity in the dynamic responses. The detection on structural sudden damage has been actively carried out in this study. The signal singularity of the acceleration responses with sudden stiffness reduction is characterized by the coefficients of continuous wavelet transform with fine scales. A detection approach based on the CWT is proposed in terms of the decomposed detail coefficients of continuous wavelet transform to detect the damage time instant and location. The Lipschitz exponent is mathematically used to estimate the local properties of certain function and is applied to reflect the damage severity. Numerical simulation using a five-story shear building under different types of excitation is carried out to assess the validity of the proposed detection approach for the building at different damage levels. The sensitivity of the damage index to the intensity and frequency range of measurement noise is also investigated. The effects of both measurement noise intensity and frequency range on the damage detection are numerically investigated.
\end{abstract}

\section{Introduction}

The wavelet transform is an extension of the traditional Fourier transform with adjustable window location and size. Wavelet analysis combines both time and frequency analysis, which allows it to zoom in on time without any loss of scale resolution. The wavelet transform has recently emerged as a promising tool for structural health monitoring and it is an ideal tool in addressing the issue of time locality of structural damages [1-4]. The earliest work of applying wavelet analysis in structural health monitoring dated back to the work of Masuda and his group in $1995[5,6]$. Faults in gear systems were detected using wavelet approaches and some results were verified by an inspection [7]. Hong et al. [8] studied the effectiveness of the wavelet transform in detecting structural cracks. In their analysis, the magnitude of the Lipschitz exponent is used as a useful indicator of the damage extent. The detection results from both numerical simulation and experiment prove the efficiency of wavelet based damage detection approaches.
The degradation of civil engineering structures may lead to a sudden stiffness reduction in a structure associated with the events such as weld fracture, column buckling, and brace breakage [9]. Such a sudden damage of stiffness in a structure will cause a discontinuity in acceleration responses and can be detected by using signal based detection approaches such as wavelet transform, empirical mode decomposition, time series, and time-frequency analysis. Hou et al. $[10,11]$ proposed a wavelet-based approach to identify the damage time instant and damage location of a simple structural model with breakage springs. Sohn et al. [12] used wavelet transform and the Holder exponent to detect the time varying nature of discontinuities. Their experimental results demonstrated that the Holder exponent could be an effective tool for identifying certain types of events that introduce discontinuities in the measured dynamic response data. The same idea for detecting sudden damage was adopted by Vincent et al. [13] and Yang et al. $[9,14]$ but using empirical mode decomposition to decompose the vibration signal to capture the signal discontinuity. In addition to the above-mentioned numerical 
studies, $\mathrm{Xu}$ and Chen [15] carried out experimental studies on the applicability of empirical mode decomposition for detecting structural damage caused by a sudden change of structural stiffness. Chen and $\mathrm{Xu}$ [16] proposed two online detection approaches for the sudden damage detection.

The sudden stiffness loss of structural components may induce the signal discontinuity in the acceleration responses close to the damage location at the damage time instant. It is reported that the time instant and location of the sudden stiffness loss can be detected by using the discrete WT. However, the severity of different sudden damage events cannot be estimated directly by the WT. In this regard, the investigation of detection on sudden damage event of building structures has been actively carried out in this study. The signal feature of the structural acceleration responses of an example building is examined. Three types of dynamic loading, sinusoidal, seismic, and impulse excitations are taken as the inputting excitations. The signal singularity of the acceleration responses with sudden stiffness reduction is characterized by the coefficients of continuous wavelet transform with fine scales. A detection approach based on the CWT is proposed in terms of the decomposed detail coefficients of continuous wavelet transform to detect the damage time instant and location. The Lipschitz exponent is mathematically used to estimate the local properties of certain function and is applied to reflect the damage severity. Numerical simulation using a five-story shear building under different types of excitation is carried out to assess the validity of the proposed detection approach for the building at different damage levels. The sensitivity of the damage index to the intensity and frequency range of measurement noise is also investigated. The effects of both measurement noise intensity and frequency range on the damage detection are numerically investigated. The made observations demonstrate that the proposed approach can accurately identify the damage events and the damage severity can be estimated by the Lipschitz exponent.

\section{Wavelet Transform}

Morlet and Grossmann initially proposed wavelet theory and Meyer developed the mathematical foundations of wavelets. The two America-based researchers Daubechies [17] and Mallat $[18,19]$ changed this by defining the connection between wavelets and digital signal processing. Wavelets have been applied to a number of areas, including data compression, image processing, and time-frequency spectral estimation. A mother wavelet $\psi(t)$ is a waveform that has limited duration and an average value of zero. Based on this mother wavelet, the wavelet kernel can be expressed by

$$
\psi_{a, b}(t)=\frac{1}{\sqrt{a}} \psi\left(\frac{t-b}{a}\right)
$$

where $a$ and $b$ are dilation and translation parameters, respectively. Both are real numbers and $a$ must be positive. Similar to the Short Time Fourier Transform, one can analyze square-integrable function $f(t)$ with wavelet transform, which decomposes a signal in the time domain into a twodimensional function in the time-scale plane $(a, b)$

$$
\begin{aligned}
W f(a, b) & =\int_{-\infty}^{+\infty} f(t) \psi_{a, b}^{*}(t) d t \\
& =\frac{1}{\sqrt{a}} \int_{-\infty}^{+\infty} f(t) \psi_{a, b}^{*}\left(\frac{t-b}{a}\right) d t,
\end{aligned}
$$

where $*$ denotes complex conjugation. The term frequency instead of scale has been used in order to aid in understanding, since a wavelet with large-scale parameter is related to low-frequency content component and vice versa. The mother wavelet $\psi(t)$ should satisfy the following admissibility condition to ensure existence of the inverse wavelet transform such as

$$
C_{\psi}=\int_{-\infty}^{+\infty} \frac{|\widehat{\psi}(\omega)|^{2}}{|\omega|} d \omega<+\infty
$$

where $\widehat{\psi}(\omega)$ is the Fourier transform of $\psi(t)$. The existence of the integral in (3) requires that

$$
\widehat{\psi}(0)=0, \quad \text { i.e., } \int_{-\infty}^{+\infty} \psi(x) d x=0 .
$$

The signal $f(t)$ can be reconstructed by an inverse wavelet transform of $W f(a, b)$ as defined by

$$
f(t)=\frac{1}{C_{\psi}} \iint_{-\infty}^{+\infty} W f(a, b) \psi_{a, b}\left(\frac{t-b}{a}\right) \frac{1}{a^{2}} d a d b .
$$

The calculating wavelet coefficients at every possible scale will generate a lot of redundant data. In some practical signal processing cases, the discrete version of the wavelet is often utilized by discretizing the dilation parameter $a$ and the translation parameter $b$. The procedure becomes much more efficient if dyadic values of $a$ and $b$ are used; that is,

$$
a=2^{j} ; \quad b=2^{j} k \quad j, k \in Z,
$$

where $Z$ is the set of positive integers. For some special choices of $\psi(t)$, the corresponding discrete wavelets can be written to constitute an orthonormal basis

$$
\psi_{j, k}(t)=2^{j / 2} \psi\left(2^{j} t-k\right), \quad j, k \in Z .
$$

Using the orthonormal basis, the wavelet expansion of a function $f(t)$ and the coefficients of the wavelet expansion are defined as

$$
\begin{gathered}
f(t)=\sum_{j} \sum_{k} \alpha_{j, k} \psi_{j, k}(t), \\
\alpha_{j, k}=\int_{-\infty}^{+\infty} f(t) \psi_{j, k}^{*}(t) d t .
\end{gathered}
$$

\section{Signal Feature due to Sudden Damage}

The signal feature due to a sudden stiffness reduction is firstly investigated by taking a five-story shear building as 
an example structure (Figure 1). The elevation of the shear building is displayed in Figure 2. The building is subject to different types of external excitations and a sudden stiffness loss occurs in the first story. The mass and horizontal stiffness of the undamaged building are uniform for all stories and the floor mass and stiffness are $1.3 \times 10^{6} \mathrm{~kg}$ and $4.0 \times 10^{9} \mathrm{~N} / \mathrm{m}$, respectively. The Rayleigh damping assumption is adopted to construct the structural damping matrix with the damping ratios in the first two vibration modes being set as 0.05 . The original building is supposed to suffer a sudden $20 \%$ stiffness reduction in the first story while the horizontal stiffness in other stories remains unchanged. The frequency reduction due to $20 \%$ stiffness reduction in the first story is small with a maximum reduction no more than $5 \%$ in the first natural frequency. The sinusoidal excitation, seismic excitation, and impulse excitation are utilized, respectively, to calculate the acceleration responses of the example building to examine the signal features due to the sudden stiffness reduction. The seismic excitation used is the first 10 second portion of the El-Centro 1940 earthquake ground acceleration (S-N component) with a peak amplitude $1.0 \mathrm{~m} / \mathrm{s}^{2}$. A sinusoidal excitation expressed by (9) with 10 second duration is assumed to act on each floor of the building

$$
f(t)=1300 \cdot \sin (4 \pi t) \quad(0 \leq t \leq 10 \mathrm{~s})(\mathrm{kN}) .
$$

An impulse excitation represented by $0.1 \mathrm{~m} / \mathrm{s}$ initial velocity is supposed to occur on the first floor of the building. The damage time instant of the building is set as $6.0 \mathrm{~s}$ for seismic excitation and sinusoidal excitation and $0.2 \mathrm{~s}$ for impulse excitation. The dynamic responses under each type of external excitation are computed by using the Newmark- $\beta$ method with a time interval of $0.002 \mathrm{~s}$. The two factors in the Newmark- $\beta$ method are selected as $\alpha=1 / 2$ and $\beta=1 / 4$.

The acceleration time histories of the first floor under seismic, sinusoidal, and impulse excitations are computed and displayed in Figure 2, respectively. To direct visual inspection of the original acceleration responses, one cannot easily find the signal feature due to the sudden damage event. Therefore, the 0.2 second portions of the acceleration responses under three external excitations are expanded to permit a detailed examination at the signal feature due to sudden stiffness loss. It is seen from Figure 2(a) that there exists a sudden jump in the original acceleration time histories at the damage time instant. The same observations made from the acceleration responses under sinusoidal and impulse excitations. It is clear that the sudden reduction of horizontal stiffness of the first floor causes a clear signal discontinuity in the acceleration response time history at the damage time instant. Since the signal discontinuity is of very high frequency, the WT can be applied to decompose the original acceleration responses and extract the signal induced by sudden damage events.

\section{Detection on Sudden Damage Event}

4.1. Description on Signal Singularity Using Lipschitz Exponent. An important property of the WT is the ability to characterize the local regularity of a certain function. To characterize singularity due to sudden stiffness loss, it is

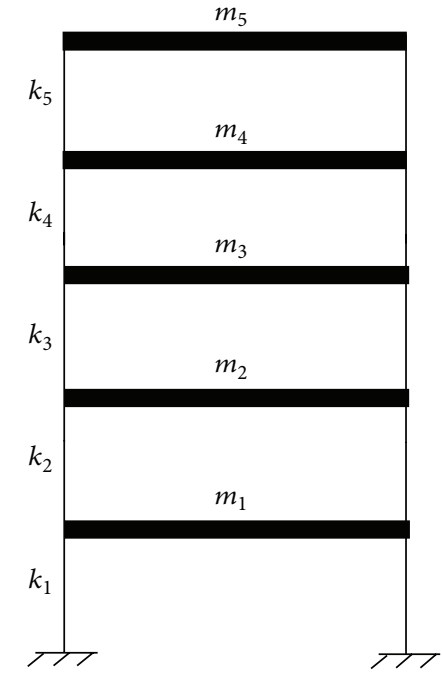

FIGURE 1: Elevation of a five-story building model.

necessary to precisely estimate the local singularity of an acceleration signal $f(t)$. Lipschitz exponents provide uniform singularity measurements over time intervals, but also at any point $v$. If the acceleration response $f(t)$ has a singularity at $v$, which means that it is not differentiable at $v$, then the Lipschitz exponent at $v$ characterizes this signal singular behaviour. An acceleration signal function $f(t)$ is said to be pointwise Lipschitz $\alpha \geq 0$ at $v$ if there exists $K>0$ and a polynomial $p_{v}$ of degree $m$ ( $m$ is the largest integer satisfying $m \leq \alpha)$ such that

$$
\begin{gathered}
f(t)=p_{v}(t)+\varepsilon_{v}(t) \\
\left|\varepsilon_{v}(t)\right|=\left|f(t)-p_{v}(t)\right| \leq K|t-v|^{\alpha} .
\end{gathered}
$$

For instance, a signal function is not differentiable at $f=v$ if $0<\alpha<1$. Therefore, the Lipschitz exponent $\alpha$ characterizes the nature of singularity at $f=v$. The traditional approach for regularity of the acceleration signal function $f(t)$ is related to the asymptotic decay of its Fourier transform. An acceleration signal function $f(t)$ is bounded and uniformly Lipschitz $\alpha$ over $\mathbb{R}$ if

$$
\int_{-\infty}^{+\infty}|\widehat{f}(\omega)|\left(1+|\omega|^{\alpha}\right) d \omega<+\infty .
$$

Equation (14) can be applied to estimate the minimum global regularity of acceleration time histories with sudden damage events. However, it is not possible to analyze the singularity of $f(t)$ at a particular point $v$ from the decay of $|\widehat{f}(\omega)|$ at high frequencies $\omega$. In contrast, since wavelets are well localized in time, the wavelet transform gives Lipschitz singularity over intervals and at points.

When the building structure suffers a sudden stiffness loss during the vibration, the extent of the signal discontinuity in acceleration time histories at the vicinity of the damage varies with different damage severities. Small damage is difficult to be identified using traditional vibration based approaches, but it still introduces some sorts of singularities 

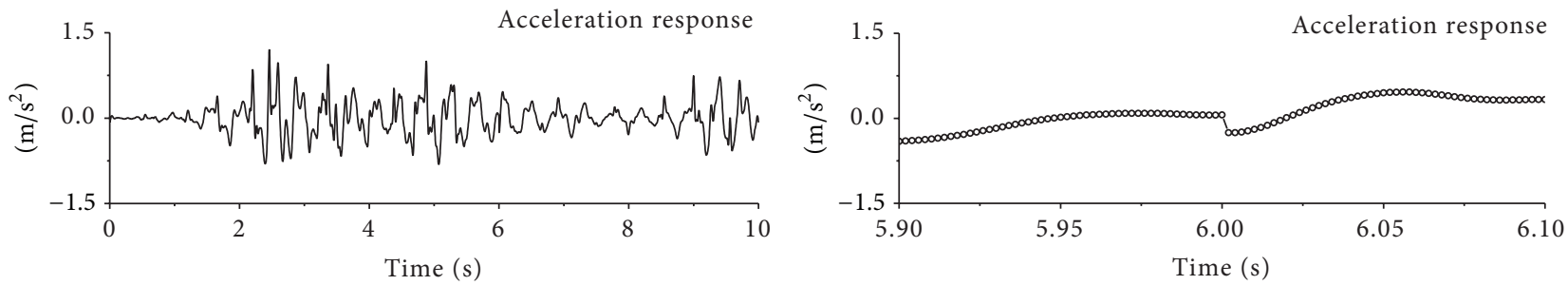

(a) Seismic excitation
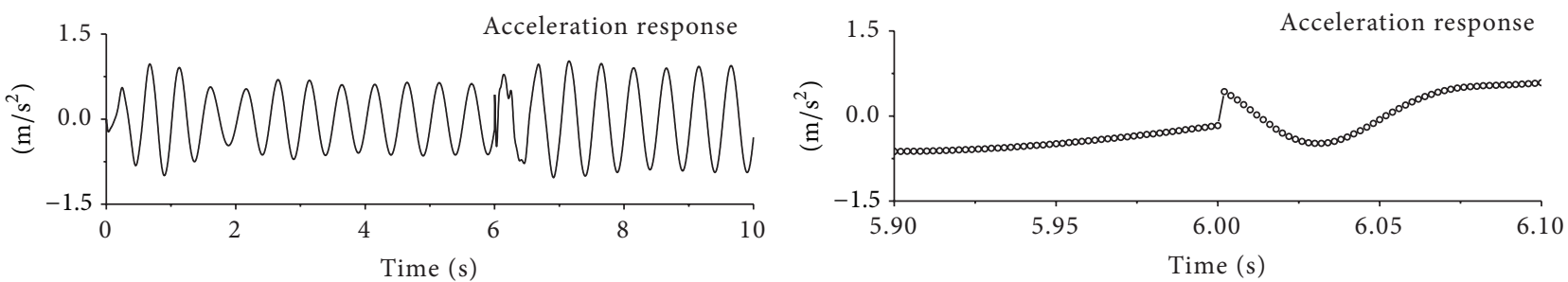

(b) Sinusoidal excitation
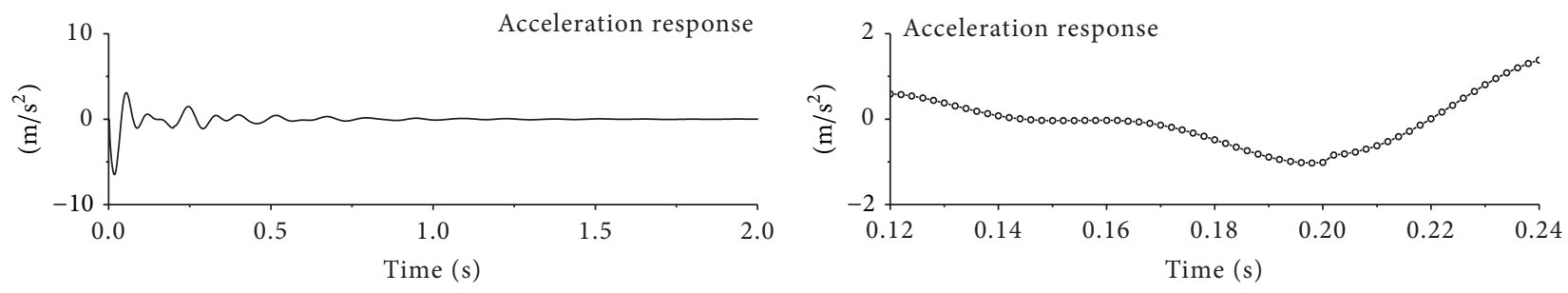

(c) Impulse excitation

FIgURE 2: Signal discontinuity due to sudden damage.

to the acceleration responses. These singularities may be characterized by using the Lipschitz exponent with the aiding of the wavelet transform. To measure the local regularity of the acceleration signal by utilizing wavelet transform and Lipschitz exponent estimation, the wavelet vanishing moment plays an important role. The wavelet transform estimates the exponent by ignoring the polynomial $p_{v}$, For this purpose, one need use a wavelet that has $n>\alpha$ vanishing moment. A wavelet $\psi(t)$ is said to have $n$ vanishing moments if it satisfies the following condition:

$$
\int_{-\infty}^{+\infty} x^{k} \psi(x) d x=0 \quad \text { for } 0 \leq k<n .
$$

The condition (15) demonstrates that the wavelet having $n$ vanishing moments is orthogonal to polynomials of up to degree $n-1$. If the wavelet transform with $n>\alpha$ is applied to (12) and consider the polynomial $p_{v}$ has degree at most $n-1$, the following equations can be obtained

$$
\begin{gathered}
W p_{v}(a, b)=\frac{1}{\sqrt{a}} \int_{-\infty}^{+\infty} p_{v}(t) \psi_{a, b}\left(\frac{t-b}{a}\right) d t=0 \\
W f(a, b)=W \varepsilon_{v}(a, b) .
\end{gathered}
$$

Therefore, the wavelet transform focuses only on a singular part of a certain function which demonstrates that CWT is suitable for detecting signal singularity due to sudden stiffness reduction. If a square-integrable function $f(t)$ is
Lipschitz $\alpha \leq n$ at $t=v$, then the asymptotic behaviour of the wavelet transform $W f$ near $t=v$ becomes [18]

$$
|W f(a, b)| \leq A a^{\alpha+1 / 2}\left(1+\left|\frac{b-v}{a}\right|^{\alpha}\right) \quad(A>0),
$$

where $A$ is a constant. If $t$ is the cone of influence of $v$ then $W f(a, b)=\left\langle f, \psi_{a, b}\right\rangle$ depends on the value of $f$ in the neighbourhood of $v$. Equation (18) can be reduced to

$$
|W f(a, b)| \leq A a^{\alpha+1 / 2} \text {. }
$$

The high-amplitude wavelet coefficients are in the cone of the influence of the singularity. Normally, it is convenient to use the logarithm form in the estimation of the Lipschitz exponent of acceleration responses at the damage time instant

$$
\log _{2}|W f(a, b)| \leq \log _{2} A+\left(\alpha+\frac{1}{2}\right) \log _{2} a \text {. }
$$

Equation (18) proves that the local Lipschitz regularity of $f$ at $t=v$ depends on the decay at fine scales of $|W f(a, b)|$ in the neighbourhood of $v$. Measuring this decay directly in the time-scale plane $(a, b)$ is not necessary. The decay of $|W f(a, b)|$ can indeed be controlled from its local modulus maxima values of the wavelet transform coefficients. The modulus maximum is used to describe any point $\left(a_{0}, b_{0}\right)$ such that $\left|W f\left(a_{0}, b\right)\right|$ is locally maximum at $b=b_{0}$

$$
\frac{\partial W f\left(a_{0}, b_{0}\right)}{\partial b}=0 \text {. }
$$


This local maxima should be a strict local maxima in either the right or left neighbourhood of $b_{0}$, to avoid having any local maxima when $\left|W f\left(a_{0}, b\right)\right|$ is constant.

4.2. Detection on Sudden Damage Event. Normally, the wavelet with at least $n$ vanishing moments is necessary to detect the $n$th derivative discontinuity of the original signal. The inspection of the acceleration response time histories subjected to various excitations demonstrates that sudden stiffness loss will cause signal discontinuity at the damage instant. Therefore, the Lipschitz exponent of the acceleration response signal should be less than 1 . To extract the Lipschitz exponent utilizing the wavelet transform, the minimum number of wavelet vanishing moment is

$$
n=1 \text {. }
$$

Signal singularity due to sudden stiffness reduction is detected by finding the abscissa where the wavelet modulus maxima converge at fine scales. The discrete wavelet transform (DWT) decomposes signal using discrete scales which cannot provide fine division especially for high frequency components of the original signal. The sudden stiffness loss will cause a high frequency damage signal in the original acceleration time history. Therefore, it is rough to utilize the DWT to obtain the Lipschitz exponent estimation and correlate the damage severity. The CWT as an alternative approach can execute continuous transform between the concerned continuous scale section which make it possible to obtain the accurate maxima line and Lipschitz exponent.

In the signal singularity detection on the acceleration responses with sudden stiffness reduction utilizing CWT, the continuity of the modulus maximum of $|W f(a, b)|$ is another factor to select mother wavelet. If a wavelet $\psi(t)$ is the $n$th derivative of Gaussian function $\theta(t)$

$$
\psi(t)=(-1)^{n} \frac{d^{n} \theta(t)}{d t^{n}} .
$$

The modulus maximum of $W f(a, b)$ belongs to a connected curve that is never interrupted for decreased scales (Mallat, 1998). Furthermore, the corresponding wavelet has $n$ vanishing moments. Following these observations, the $n$ can be taken as 2 in the detection on sudden stiffness reduction and the mother wavelet for CWT is written as

$$
\psi(t)=\frac{d^{2} \theta(t)}{d t^{2}} .
$$

The wavelet in (20) is usually referred to as the Marr or Mexican hat wavelet and has the following explicit expression:

$$
\psi(t)=\frac{2}{\sqrt{3 \sigma}} \pi^{-1 / 4}\left(\frac{t^{2}}{\sigma^{2}}-1\right) \exp \left(-\frac{t^{2}}{2 \sigma^{2}}\right) .
$$

The graph $-\psi(t)$ for $\sigma=1$ is shown in Figure 3 .

\section{Case Study}

5.1. Damage Time Instant. To examine the validity of the proposed detection approach for identifying sudden damage

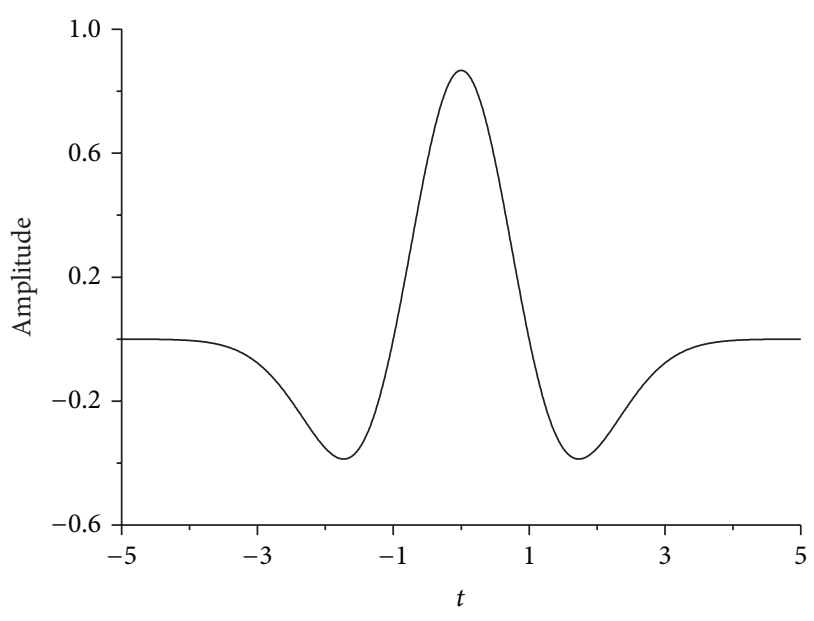

Figure 3: The Marr wavelet $(-\psi(t)$ and $\sigma=1)$.

events, the acceleration responses of the example building subjected to the seismic excitation, sinusoidal excitation, and impulse excitation are computed, respectively. The building is subject to a $20 \%$ sudden stiffness reduction at times $6.0 \mathrm{~s}$, $6.0 \mathrm{~s}$, and $0.2 \mathrm{~s}$ in the first story of the building under seismic excitation, sinusoidal excitation, and impulse excitation, respectively. The time step used in the computation is 0.002 second. Figure 4 displays the time-scale-coefficient plot for $20 \%$ sudden stiffness reduction, under sinusoidal, seismic, and impulse excitations, respectively. It can be seen from Figure 4 that the CWT coefficients of the first floor using Marr wavelet are very large only at time $t=6.0$ second under small decomposition scale 0.5 , which is exactly the time instant of the sudden damage event. The CWT coefficients of the first floor at all other time instants are very small, so that the CWT coefficients at time $t=6.0 \mathrm{~s}$ looks like a spike to indicate the occurrence time of the sudden damage event.

5.2. Selection of Decomposition Scale. Regarding the building excited by El Centro ground motion, CWT coefficients using large decomposition scales, such as 3.0, fail to detect damage instant while the counterparts using fine scales successfully capture the damage event. If the decomposition scales are relatively small, such as 0.5 , CWT coefficients can depict the high frequency components of the original signal and the peak of modulus maxims can be observed at damage instant based on a fine scale interval to depict the damage event. With the increase of decomposition scales $(>1.0)$, however, the frequency components reflected by modulus maxima of CWT coefficients gradually decrease. Thus, middle and high frequency components in the original acceleration responses form some modulus peaks, which make it difficult to detect the sudden damage event.

To examine the structural acceleration responses induced by impulse excitation, one can find that only the CWT coefficients with fine decomposition scale can detect the damage instant. In reality, the sudden stiffness reduction will cause a discontinuity in acceleration responses at damage instant and induce high frequency components into 

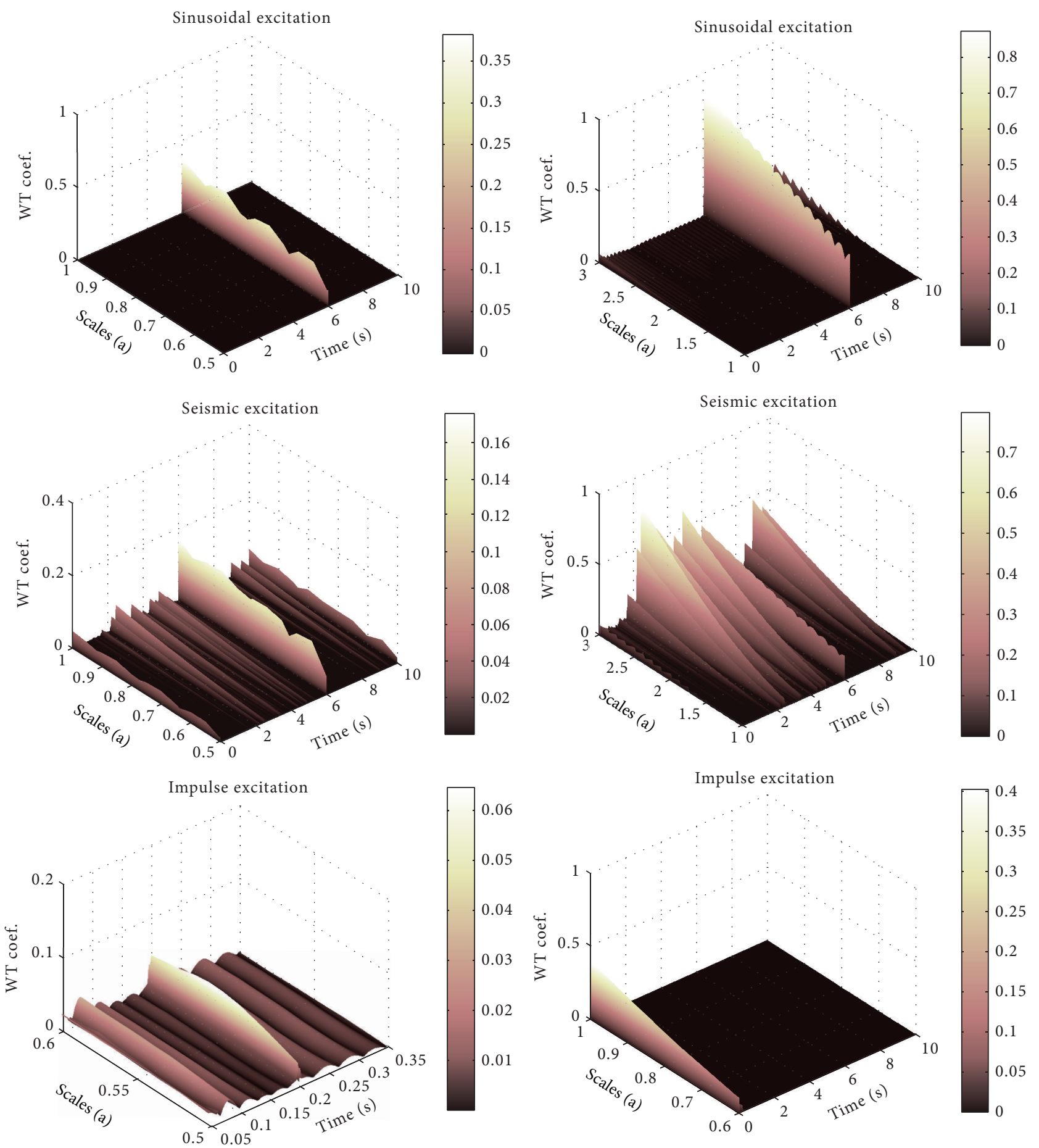

Figure 4: Damage detection under different decomposition scales.

the original response signals. Therefore, one can extract the high frequency components from original acceleration responses using the WT and detect the sudden damage event. The frequency components of acceleration responses of the building subjected to sinusoidal excitation are quite simple and the high frequency signal induced by sudden damage is quite different from other signal components. The CWT coefficients can easily detect the signal singularity and damage event even using coarse decomposition scales. As far as the seismic excited damage building is concerned, the acceleration responses contain more high frequency components than those induced by the sinusoidal excitation. The distinguishing ability in the time-frequency domain under large scale is coarse and it is difficult to capture the sudden damage event under seismic excitations. Under large decomposition scales, it is impossible to distinguish the 

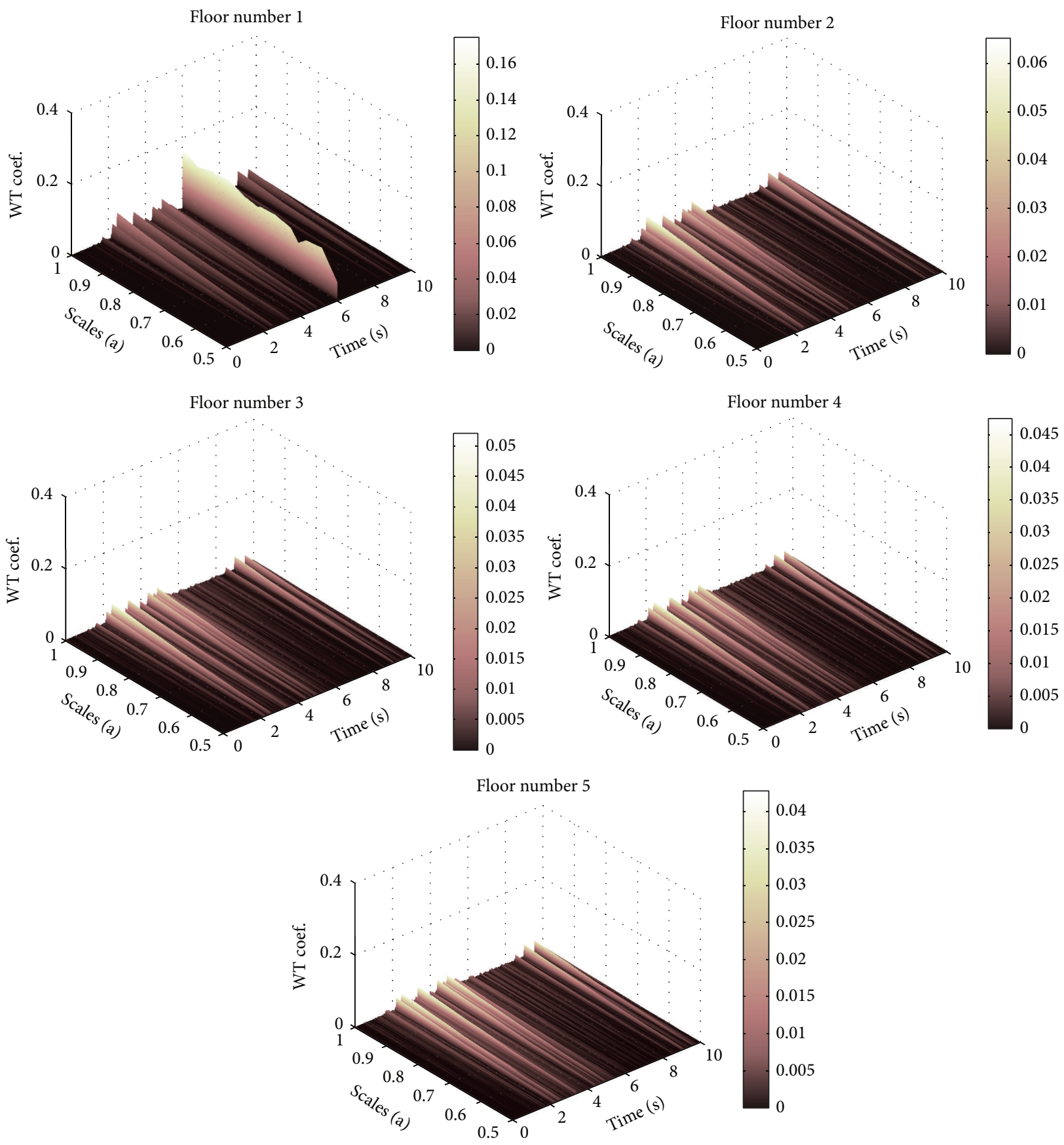

FIGURE 5: Damage detection for each floor under seismic excitation.

modulus maxima due to the sudden damage event and the damage detection is not satisfactory.

5.3. Damage Location. Figure 5 shows the variations of CWT coefficients with time for each floor of the building under the seismic excitation. It is seen that the modulus of CWT coefficients of the first floor is very large only at time $t=$ 6.0 second with fine decomposition scales, which is exactly the moment for the $20 \%$ sudden stiffness reduction. The variations of CWT coefficients of the first floor are compared with those of the second, third, fourth, and fifth floors of the building under small decomposition scale 0.5 . The sharp spike appears clearly only at the first floor, and no sharp spike emerges in other floors. Therefore, the damage location can be easily identified at the first story of the building by checking the distribution of spikes along the height of the building.

The modulus maxima lines for each floor under sinusoidal and impulse excitations with $20 \%$ stiffness loss are also investigated, respectively, and the results are not displayed for space limitation. Again, the modulus maximum lines 

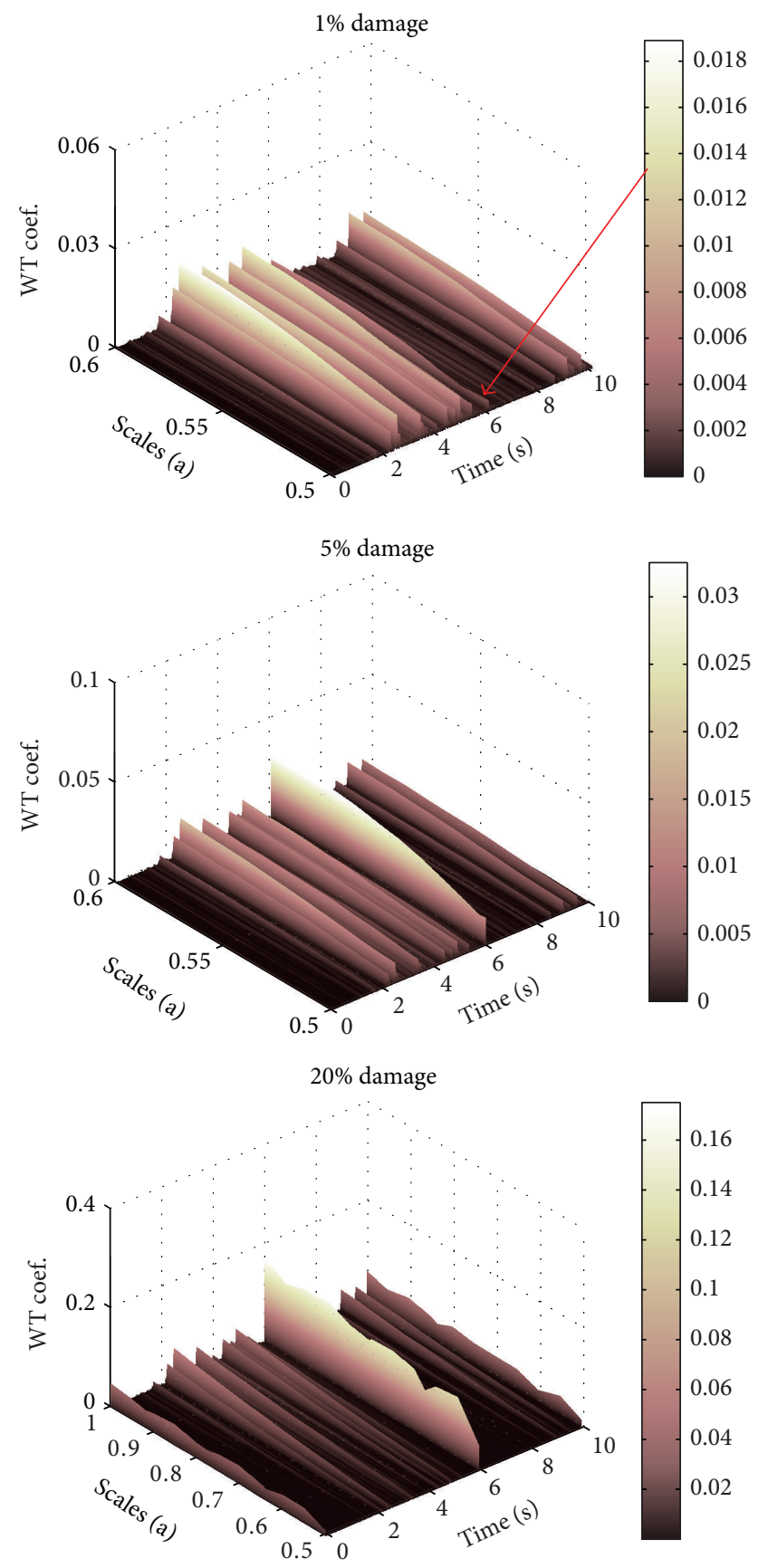
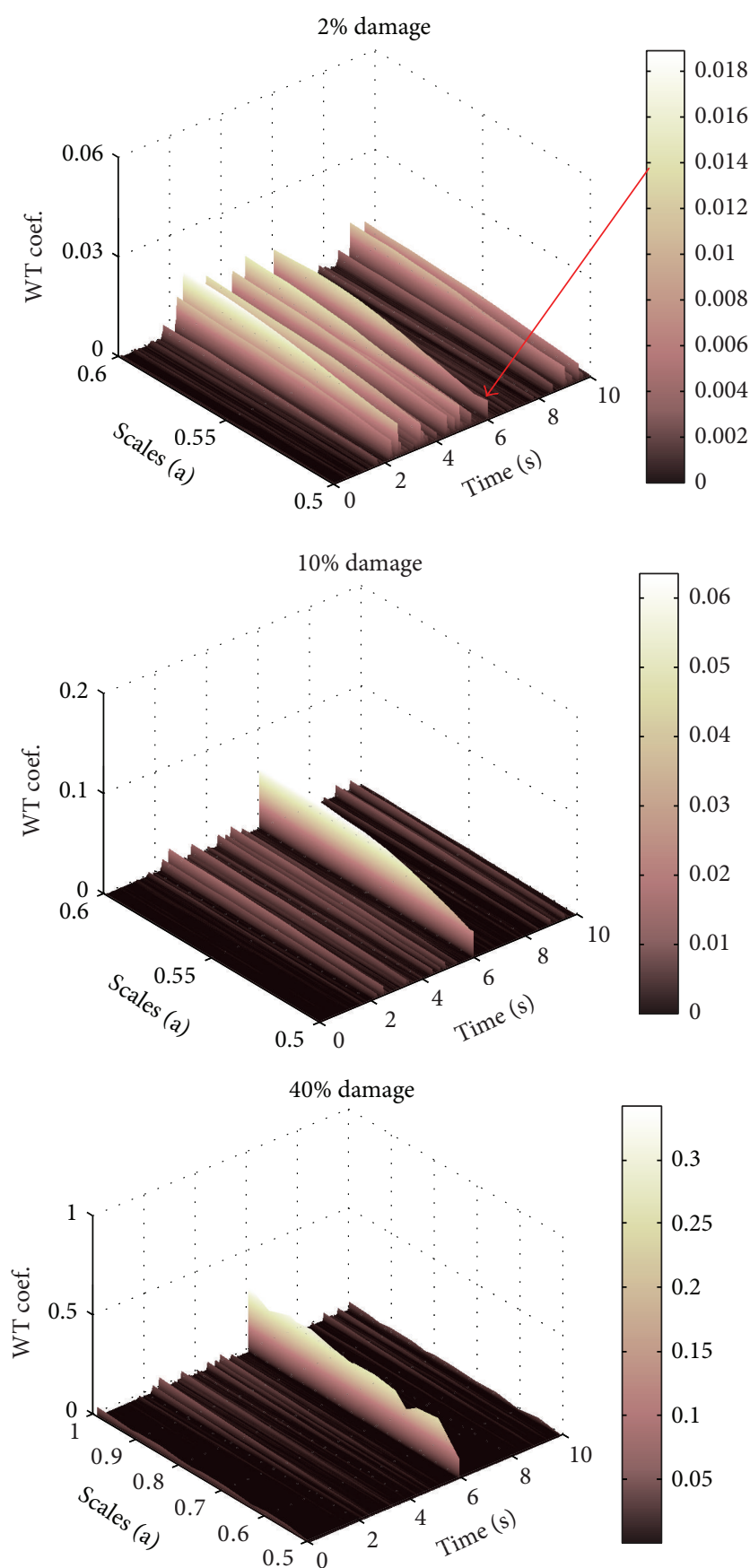

FIGURE 6: Detection results for various severities under seismic excitation.

of CWT coefficients appear only at the moment of sudden stiffness reduction at the first floor. Thus, the damage location can be easily captured from the observed maxima line and its distribution along the height of the building. As far as the impulse excited building is concerned, the CWT based detection approach may not give satisfactory results for the building with small damage event ( $2 \%$ sudden stiffness reduction). This is because the signal fluctuates significantly and the energy of damage signal is quite weak.

5.4. Damage Severity. The parametric study is carried out in this section to investigate the sensitivity of CWT coefficients to damage severity so as to examine the validity of the proposed damage detection approach. The first floor of the example building is supposed to suffer different levels of sudden stiffness reduction but the damage time instants remain unchanged. The CWT coefficients of the first floor of the building subjected to the seismic excitation are displayed in Figure 6 for the sudden stiffness reduction from $1 \%$ to $40 \%$. It can be seen that if the damage severity is no less than $5 \%$, the proposed approach can easily capture the damage features without considering noise contamination with fine decomposition scale. The magnitude of the sharp spike also 

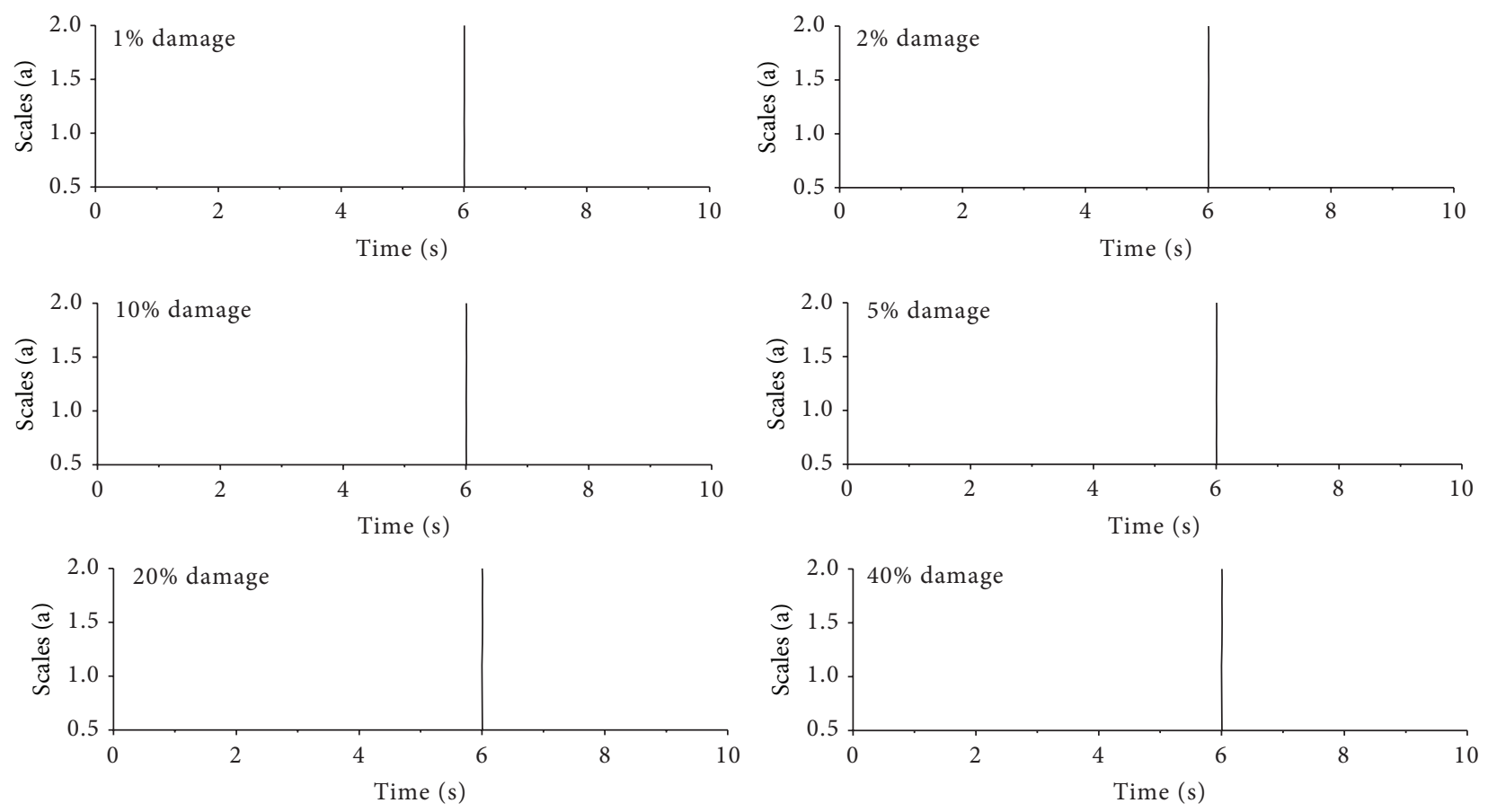

Figure 7: Modulus maxima line for various severities under seismic excitation.

TABLE 1: Variations of Lipschitz exponent with damage severity without noise.

\begin{tabular}{lcccccc}
\hline Damage severity & $1 \%$ & $2 \%$ & $5 \%$ & $10 \%$ & $20 \%$ & $40 \%$ \\
\hline Seismic excitation & 0.9901 & 0.8008 & 0.6092 & 0.5146 & 0.4558 & 0.4086 \\
Sinusoidal excitation & 0.9411 & 0.8202 & 0.6925 & 0.5788 & 0.5144 & 0.4787 \\
Impulse excitation & 0.9991 & 0.9734 & 0.9317 & 0.8863 & 0.7005 & 0.5276 \\
\hline
\end{tabular}

increases with the increasing damage severity. Regarding the small damage events, such as $1 \%$ and $2 \%$ stiffness reduction, small damage signal energy induces the quick reduction of modulus maxima at damage instant. To carefully compare the modulus maxima line of the building with minor damage, a relative larger spike still can be found at damage instant to depict the sudden damage event. Similar observations can be made from the building subject to sinusoidal excitation. For the building under impulse excitation, however, the proposed approach may not provide satisfactory detection quality for the building with very small damage event (damage severity no more than 5\%).

Figure 7 displays the modulus maxima line for various damage severities under seismic excitation. It is observed that the damage instant can be indicated without any time excursion even for small damage severities under large decomposition scales. Displayed in Figure 8 is the decay behaviour along the modulus maxima line for the building subjected to $20 \%$ sudden stiffness reduction under seismic excitation. It is clear that the modulus maxima become larger with the increasing decomposition scale. The Lipschitz exponent is estimated as 0.4558 by using the well-known linear regression technique.

It is reported by Mallat [18] that the Lipschitz exponents become smaller with the increasing extents of the singularity in original signals. To investigate the relation between the damage extent and the Lipschitz exponent, the different damage severity under sinusoidal, seismic, and impulse excitations are examined, respectively, by repeating the same estimation procedure and the results are shown in Figure 9. The variations of Lipschitz exponent with damage severity without noise are listed in Table 1 . It is found that the magnitude of the Lipschitz exponents gradually decreases with the increasing damage severities. The relationship between Lipschitz exponent and damage severity is different if the building is subjected to different dynamic excitations. The relationship between Lipschitz exponent and damage severity under impulse excitations is closely linear while the counterparts under sinusoidal and seismic excitations seem to be decaying exponent curves.

5.5. Selection of Mother Wavelet. To examine the feasibility of the proposed detection approach based on CWT and Lipschitz exponent, the first floor of the five-story building is supposed to suffer $20 \%$ sudden stiffness reduction but the sudden reduction occurs at the same time. The detection quality substantially depends on the characteristics of the mother wavelet such as wavelet vanishing moments and supporting length in the time domain. Thus, six different mother wavelets, Haar, Meyer, Morlet, Symlet-2, Daubechie-2, and 

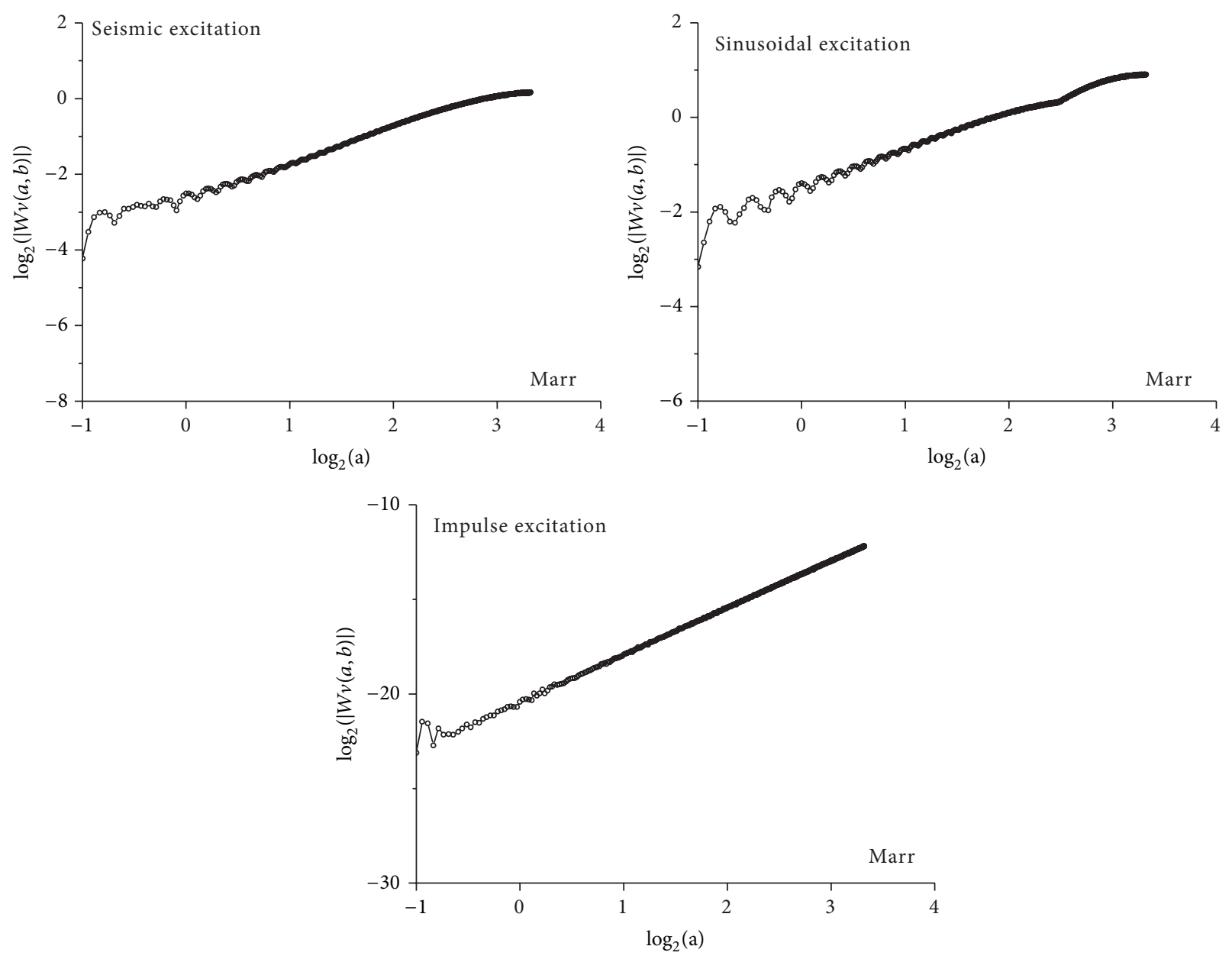

FIgURE 8: Decay behaviour along the modulus maxima line under seismic excitation.

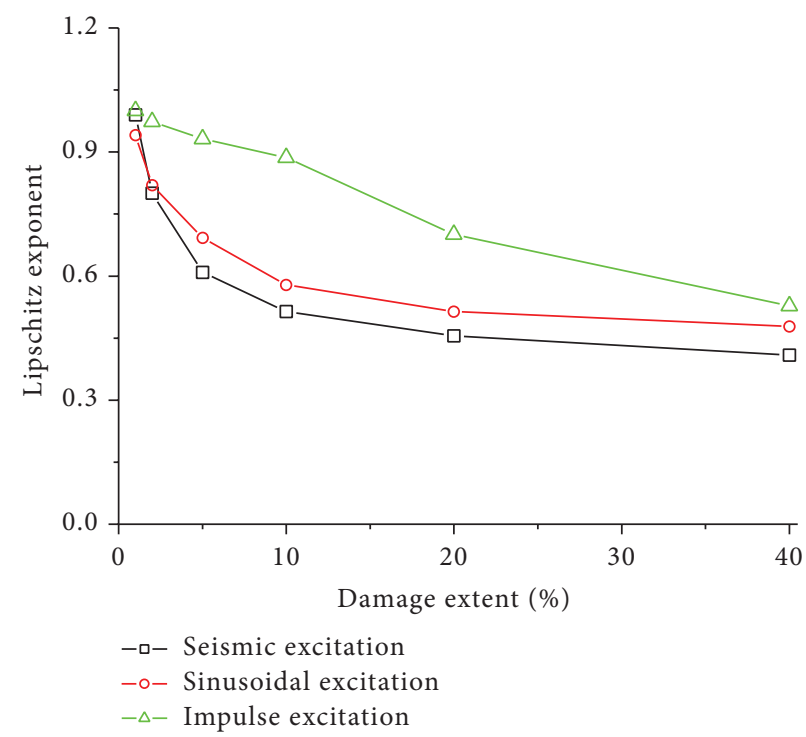

FIGURE 9: Decay behaviour along the modulus maximum line under seismic excitation. 

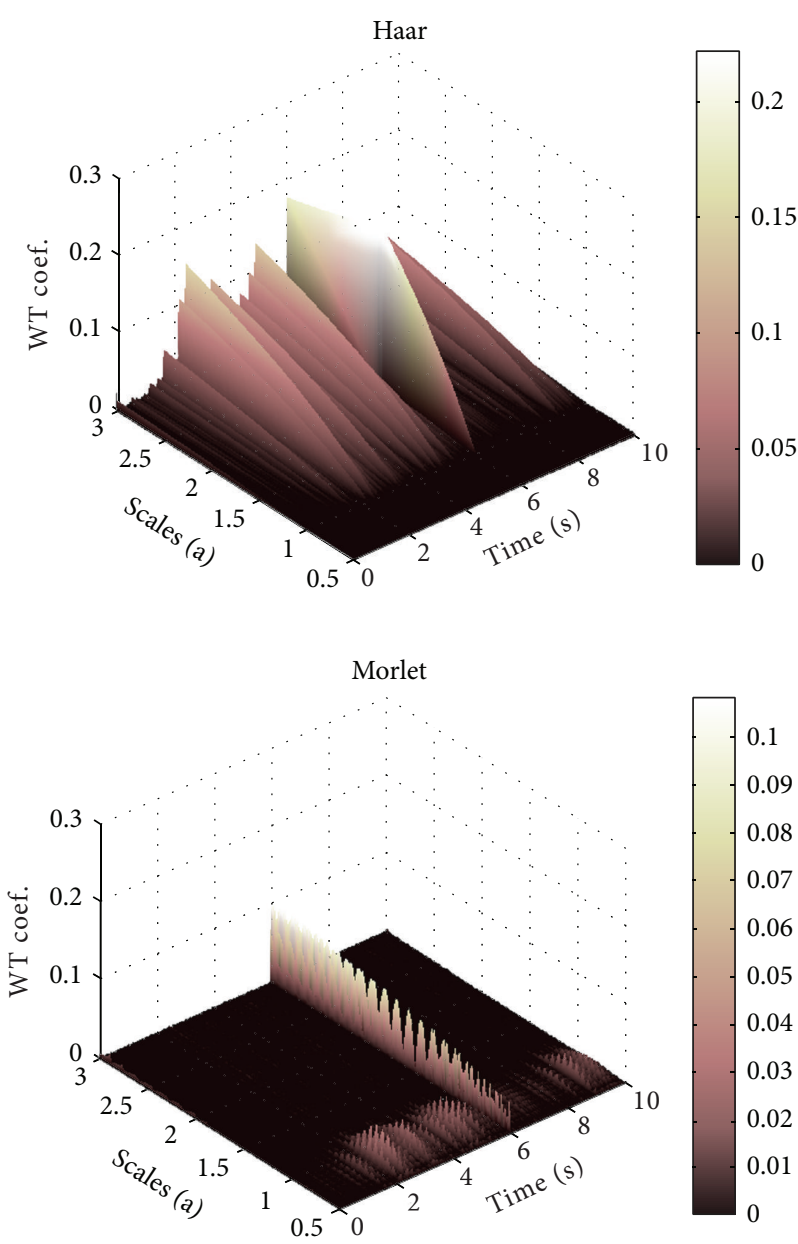

$\mathrm{db} 2$
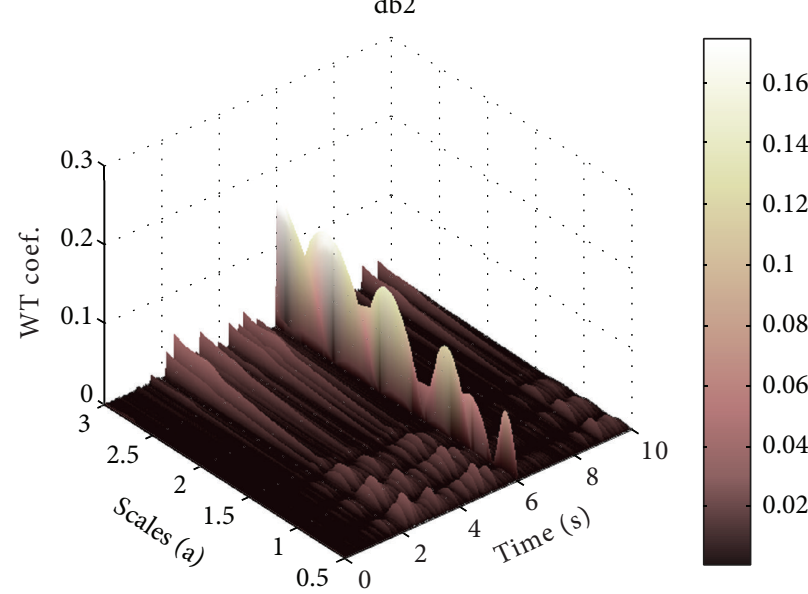

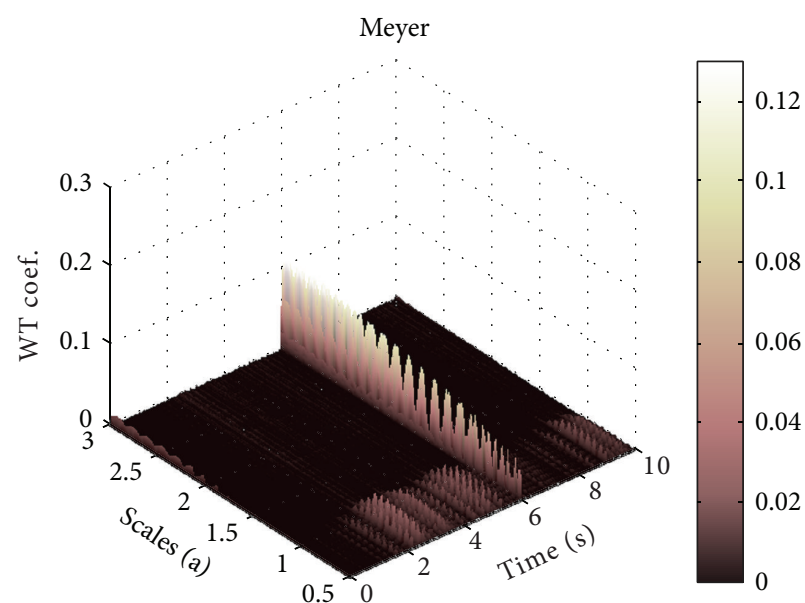

Symlets-2

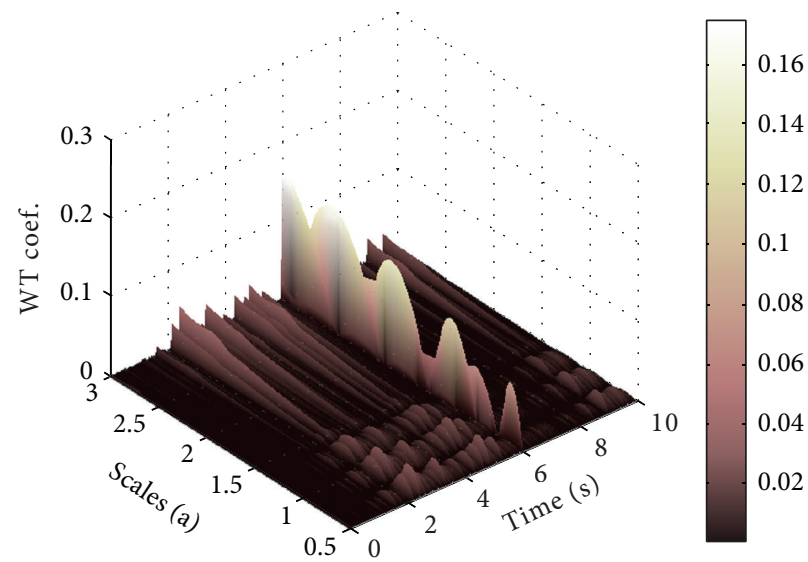

Coiflets-2

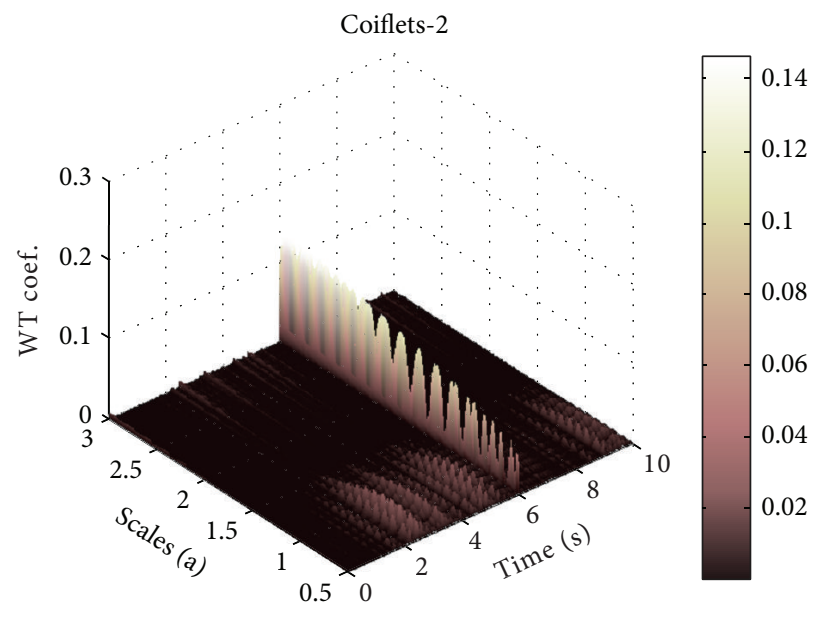

FIGURE 10: Detection results under seismic excitation using different mother wavelets.

Coiflet-2, are utilized to examine the effects of properties of mother wavelets on the detection on the structural sudden damage events. The vanishing moments of the Haar, Meyer, Morlet, Symlet, Daubechie-2, and Coiflet-2 wavelets are 1, indefinite, indefinite, 2,2 , and 2 , respectively. The damage detection results under seismic excitation using different mother wavelets are shown in Figure 10. It can be seen that no matter which mother wavelet is used, the CWT coefficients of the first floor under small decomposition scale $(<1.0)$ are very large only at time $t=6.0$ second, which is exactly the moment when the stiffness of the first story is suddenly reduced by $20 \%$. The damage indices of the first floor at all other time instants are small so that the CWT coefficients at time $t=6.0$ second look like a spike to indicate the damage events. This is 

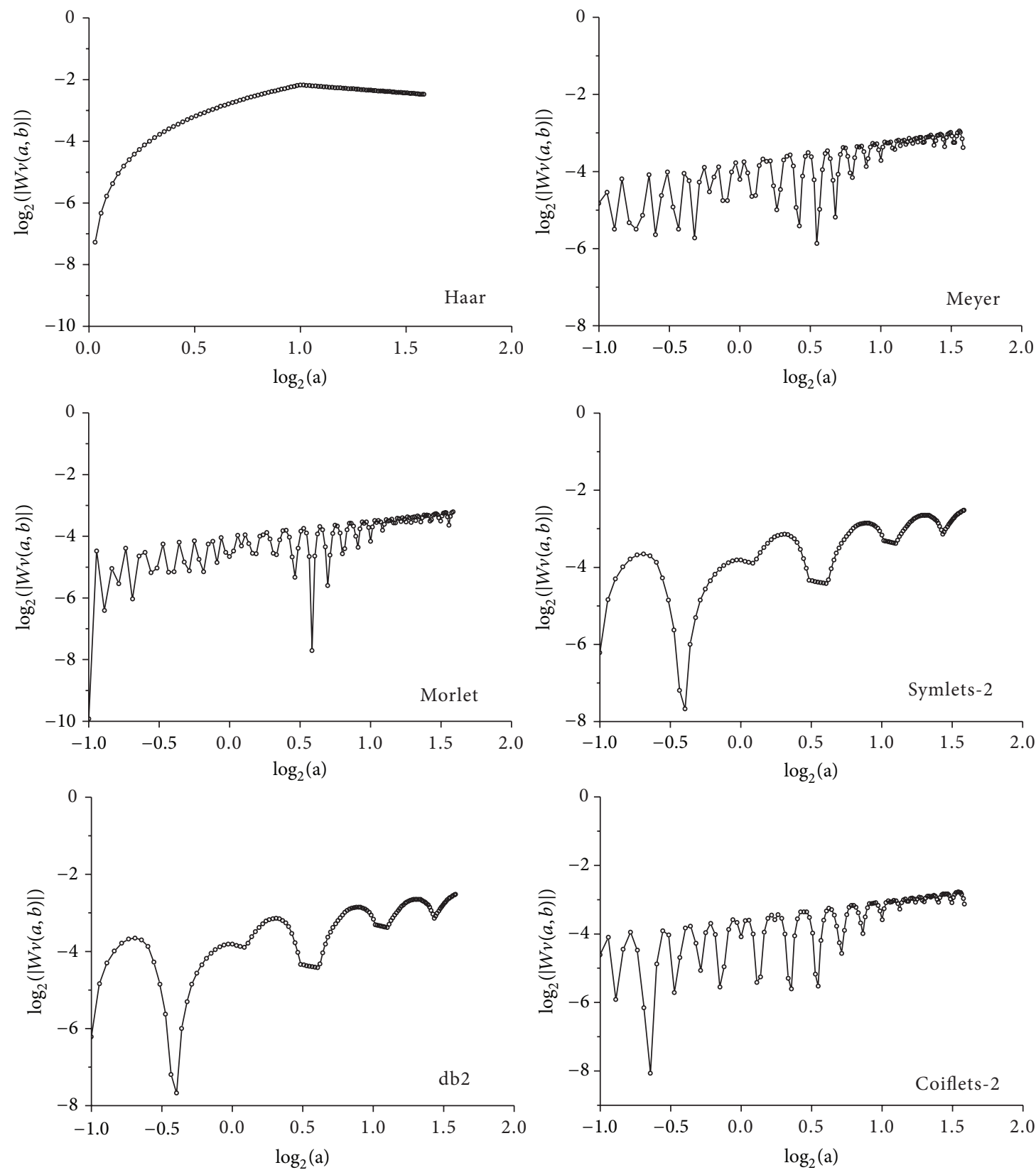

FIGURE 11: Decay behaviour along the modulus maxima line using different mother wavelets.

because all the six mother wavelets have a vanishing moment larger than one to depict the damage events as required by (22).

It is also found that the CWT based approach using all six mother wavelets can accurately detect the damage time instant of the building subjected to sinusoidal excitation and impulse excitation. The decay behaviour along the modulus maxima line for the building subjected to $20 \%$ sudden stiffness reduction under seismic excitation is displayed in Figure 11. It is seen that the modulus maxima using other mother wavelets fluctuate to great extents in comparison with that made by using Marr wavelet. Therefore, it is unreasonable and difficult to obtain the estimation of the Lipschitz exponent through the linear regression technique.

As discussed above, the vanishing moment of a mother wavelet plays an important role in the detection of signal singularity. To this end, the effects of vanishing moments on the detection quality are investigated by using Daubechie and Coiflet wavelets. Figures 12 and 13 display the variations of WT coefficients and the decay behaviour along the modulus maxima line with different vanishing moments. The made observations indicate that the damage time instant and location can be detected with increasing of vanishing moments. However, it is seen from Figure 11 that the fluctuation of 

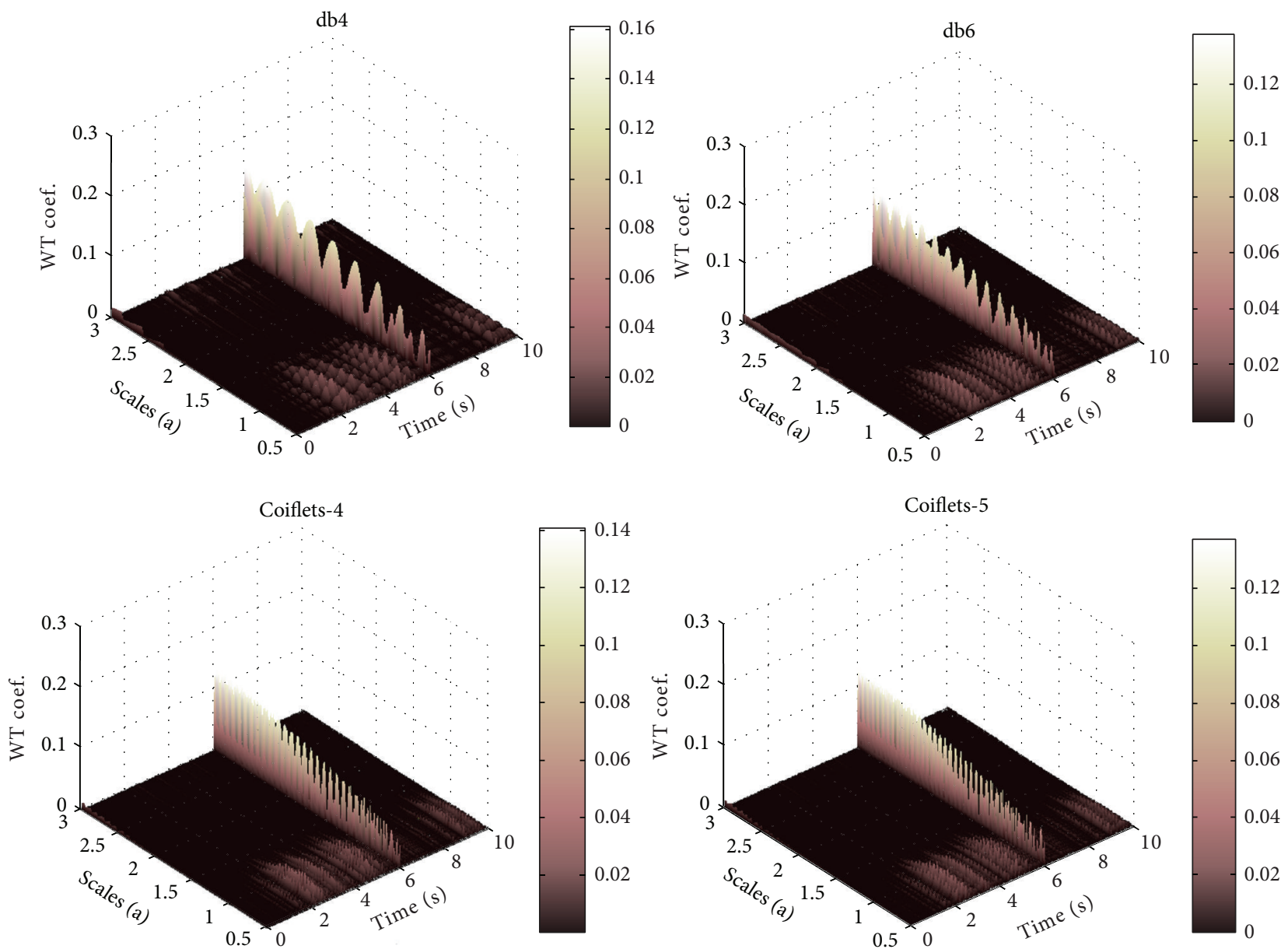

FIGURE 12: Variations of WT coefficients with different vanishing moments.

modulus maxima lines cannot be improved with the increasing vanishing moments of wavelets. Therefore, the detection quality of the CWT based approach depends on the vanishing moment and type of selected mother wavelet.

5.6. Effects of Signal Noise. To effect of the noise contamination is a practical issue need to be addressed before applying the proposed approach to health monitoring and damage detection of real structures. Yang et al. (2004) reported that the damage spike identified could be weakened by measurement noise, and strong measurement noise could lead to the failure of damage detection. Hong et al. [8] found that the damage spike identified from the wavelet transform coefficients could be weakened by the noise contamination. Taking into consideration that the sudden damage event introduces a high frequency component to acceleration responses of a structure, the effects of both measurement noise intensity and frequency range on the damage detection are investigated in this study. The measurement noise in structural responses is assumed to be a random white noise. Three frequency ranges are considered: (1) white noise with frequency range from 0 to $50 \mathrm{~Hz}$; (2) white noise with frequency range from 0 to $100 \mathrm{~Hz}$; and (3) white noise with frequency range from 0 to $250 \mathrm{~Hz}$. The measurement noise intensity is defined as

$$
\text { Noise intensity }=\frac{\text { RMS (noise) }}{\text { RMS }(\text { signal })} \times 100 \% \text {. }
$$

Displayed in Figure 14 are damage detection results using the contaminated acceleration responses at the first floor under the seismic excitation. The noises are introduced with two noise intensities and three noise frequency ranges described above. The sudden stiffness reduction in the first story of the building is $20 \%$. It is seen that the CWT based detection approach can still identify the damage time instant from the contaminated acceleration responses at the first floor for the designated two noise intensities and three noise frequency ranges. Furthermore, the spatial distribution of CWT coefficients along the height of the building can indicate the damage location from the acceleration responses with noise contamination. To check the CWT coefficients shown in Figure 14(a), one can easily find that the noise with low frequency components will not remarkably change the modulus maxima of CWT coefficients under small decomposition scales. With the increase of noise signals with high frequency 

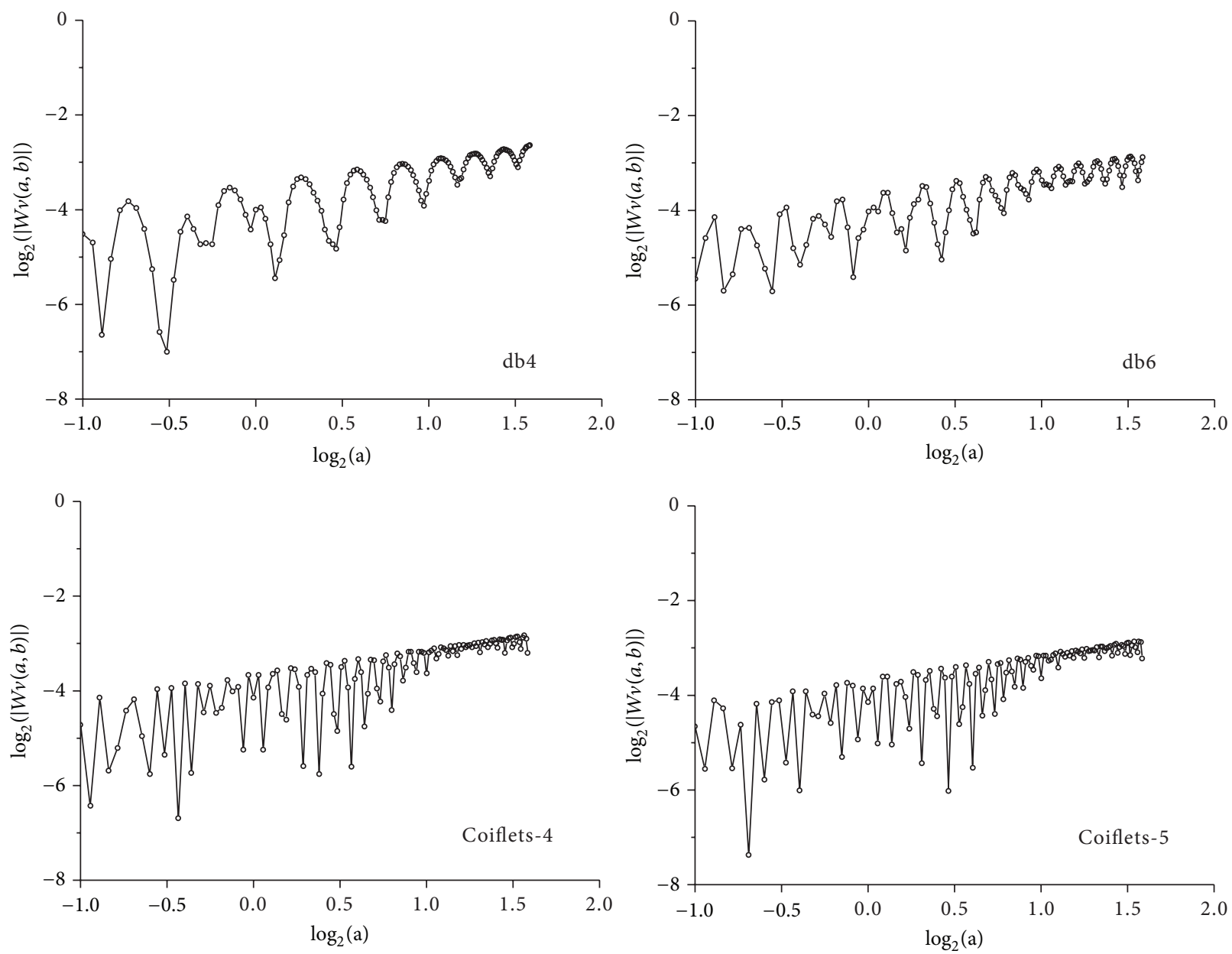

FIGURE 13: Decay behaviour along the modulus maxima line with different vanishing moments.

components, more small spikes of modulus maxima of CWT coefficients can be observed in the small decomposition scale section. In addition, the increase of noise intensity may induce the small spikes to the modulus maxima of CWT coefficients. Similar observations can be made from the detection observations of the example building subjected to the sinusoidal and impulse excitations, respectively, as shown in Figure 15. In the impulse excitation case, however, the proposed approach fails to identify the damage time instant and damage location when the noise frequency range is from 0 to $250 \mathrm{~Hz}$ and the noise intensity is $5 \%$.

It is worth examining the effects of noise contamination on the magnitude of Lipschitz exponent. The effects of measurement noise on the magnitude of the Lipschitz exponent under seismic excitation are assessed and the results are listed in Table 2. It can be seen that as long as the damage event can be identified, the magnitude of Lipschitz exponent slightly reduces with increase of noise levels and three noise frequency ranges. This indicates that the effect of measurement noise on the magnitude of Lipschitz exponent is small. The further numerical simulation indicates that the damage can be effectively identified for $20 \%$ sudden stiffness reduction even with $40 \%$ noise intensity as long as the noise frequency range is not higher than $50 \mathrm{~Hz}$. However, if the noise frequency range is from 0 to $250 \mathrm{~Hz}$, the reliability of damage detection using the proposed approach deteriorates with the increase of noise intensity. The estimation of Lipschitz exponents for different noise intensity and frequency range under sinusoidal and impulse excitation is carried out. The variations of noise intensities with Lipschitz exponents with noise frequency range $0 \sim 250 \mathrm{~Hz}$ are displayed in Figure 16. Similar observations to those under seismic excitation can be made, which agrees with the theoretical analysis of signal process (Mallat, 1998). This is because large singularity can be observed at the damage instant with noise contamination, which will further decrease the value of the Lipschitz exponent to some extent.

\section{Concluding Remarks}

The investigation of detection on sudden damage event of building structures has been carried out in this study. The signal feature of the structural acceleration responses of an example building subjected to sinusoidal, seismic, and impulse excitations due to sudden stiffness reduction is examined. The local signal regularity of the acceleration responses 

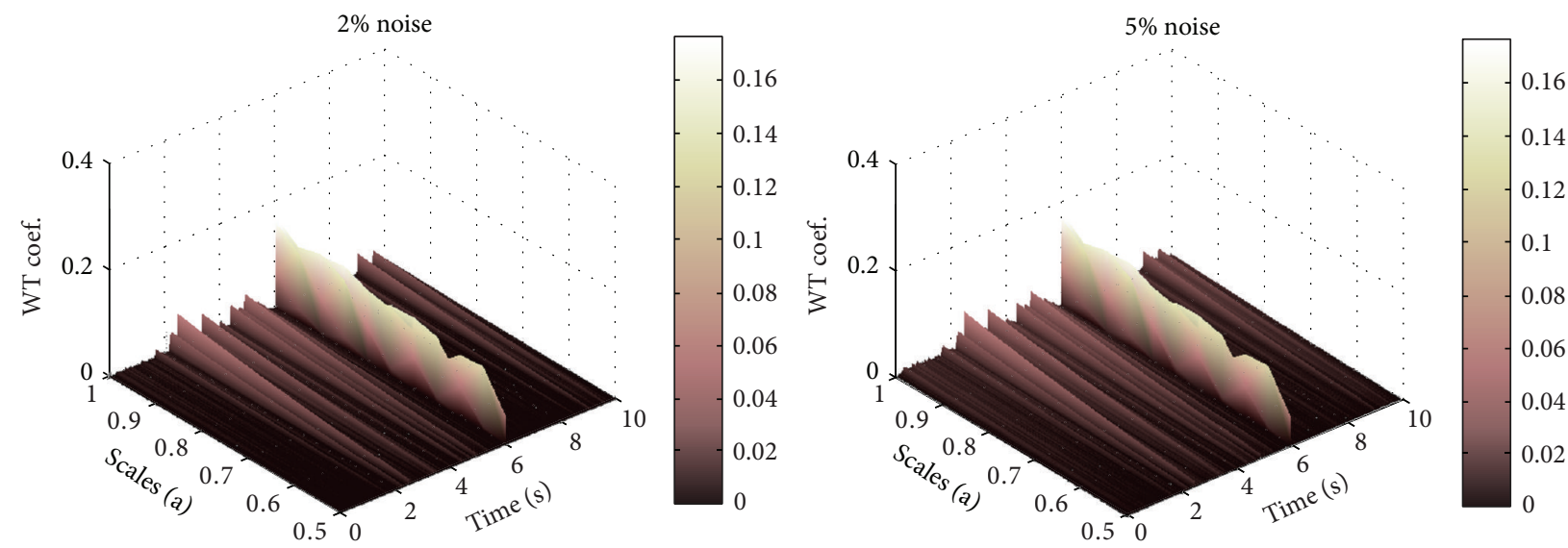

(a) Noise frequency range $0 \sim 50 \mathrm{~Hz}$
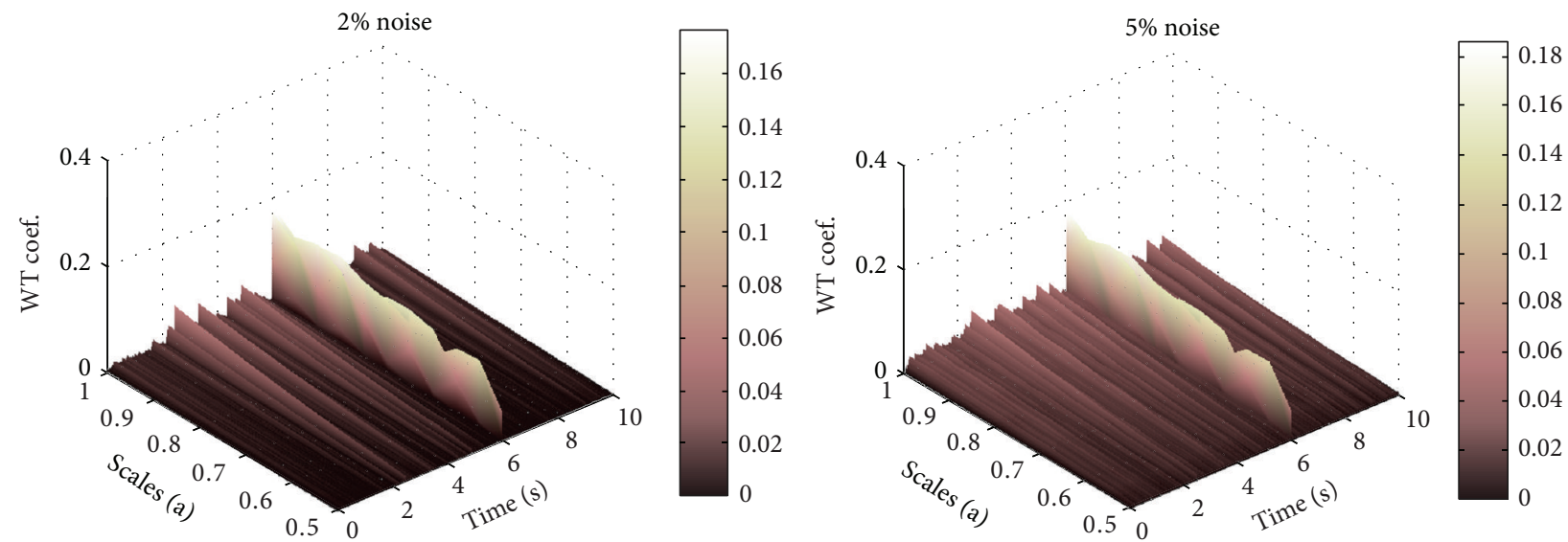

(b) Noise frequency range $0 \sim 100 \mathrm{~Hz}$
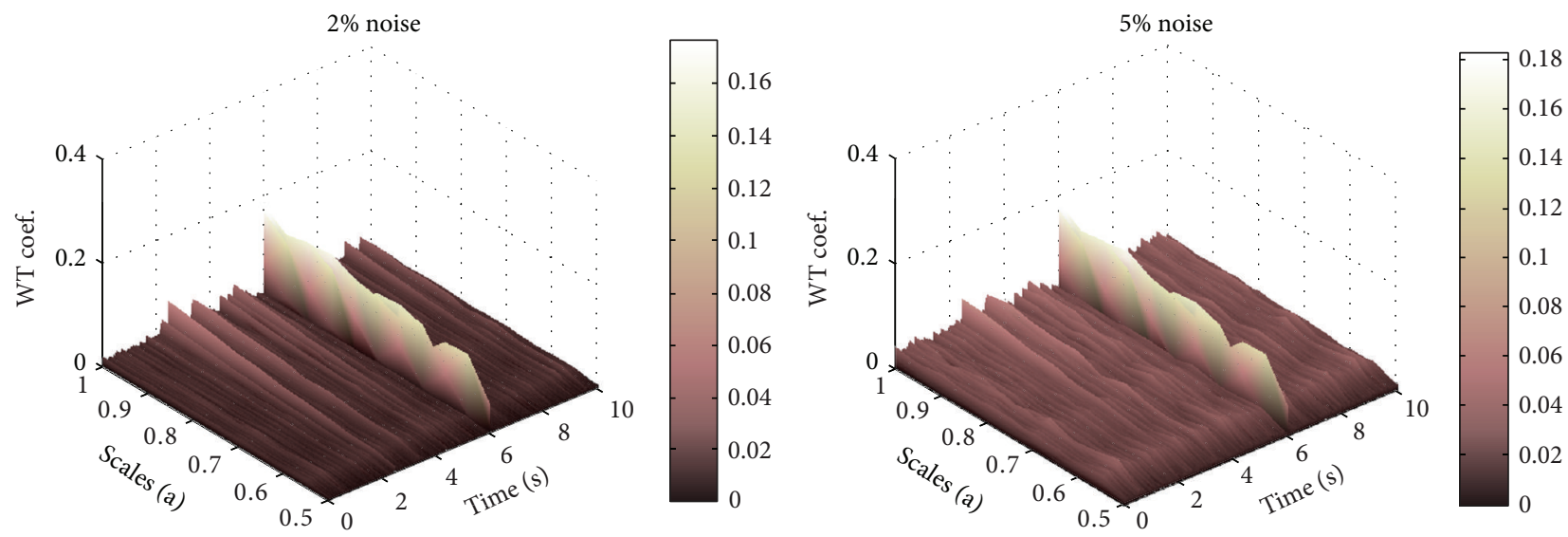

(c) Noise frequency range $0 \sim 250 \mathrm{~Hz}$

FIGURE 14: Detection results from contaminated acceleration responses (seismic excitation).

is characterized by the decay of the wavelet transform amplitude across scales. Singularities can be detected by the continuous wavelet transform local maxima at fine scales. In this regard, a detection approach based on the CWT is proposed in terms of the decomposed detail coefficients of continuous wavelet transform to detect the damage time instant and location. The Lipschitz exponent is mathematically used to estimate the local properties of certain function and is applied to reflect the damage severity.

Extensive numerical simulations have been performed on a five-story shear building to assess the performance of the detection approach based on CWT and Lipschitz exponent with and without noise contamination. The made observations indicate that the detection approach proposed 

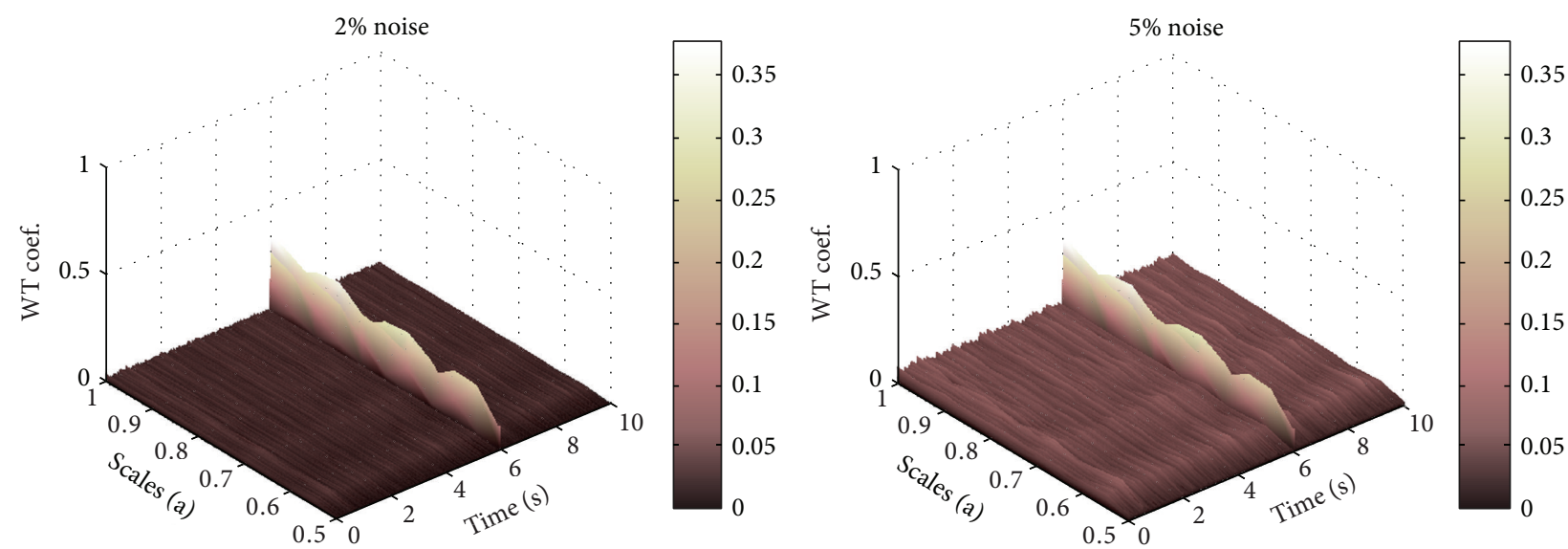

(a) Sinusoidal excitation
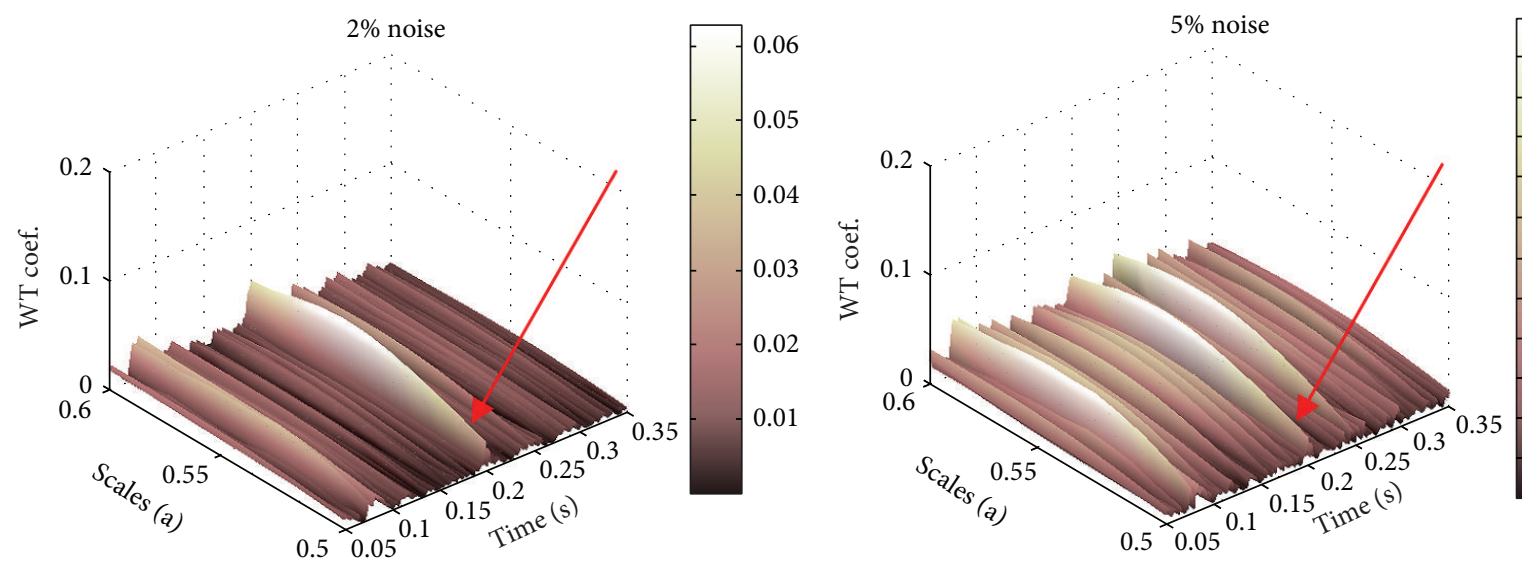

0.055 0.05 0.045 0.04 0.035

0.03 0.025 0.02 0.015 0.01 0.005

(b) Impulse excitation

FIGURE 15: Detection results from contaminated acceleration responses (noise frequency range $0 \sim 250 \mathrm{~Hz}$ ).

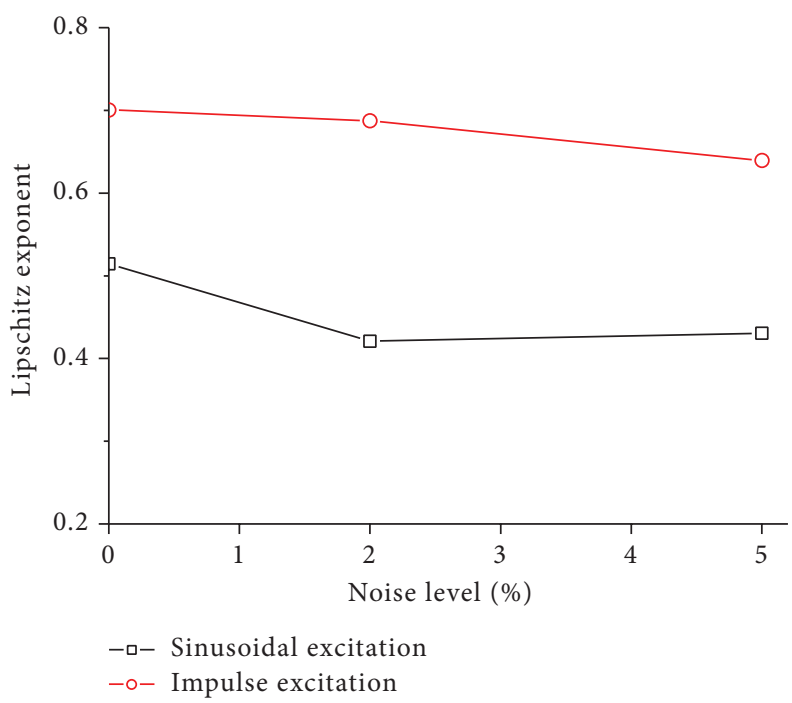

FIGURE 16: Variations of Lipschitz exponent with noise intensity under sinusoidal and impulse excitation. 
TABLE 2: Noise effects on Lipschitz exponent (seismic excitation).

\begin{tabular}{lccc}
\hline Noise level & \multicolumn{3}{c}{ Noise frequency range } \\
& $0 \sim 50 \mathrm{~Hz}$ & $0 \sim 100 \mathrm{~Hz}$ & $0 \sim 250 \mathrm{~Hz}$ \\
\hline No noise & 0.3252 & 0.3252 & 0.3252 \\
$2 \%$ noise & 0.3249 & 0.3244 & 0.319 \\
$5 \%$ noise & 0.3248 & 0.3204 & 0.3093 \\
\hline
\end{tabular}

in this study can accurately identify the damage time instant and damage location due to a sudden stiffness reduction in terms of the occurrence time and spatial distribution of coefficient spikes of the CWT. The relationship between Lipschitz exponent and damage severity is different when subjected to different dynamic excitations. The magnitudes of the Lipschitz exponents decrease with the increasing damage severity. The detection quality on the sudden damage even is satisfactory if the noise frequency range is limited. If the noise frequency range is wide enough, the reliability of damage detection quality using the proposed approach gradually decreases with the increase of noise intensity.

\section{Conflict of Interests}

The authors declare that there is no conflict of interests regarding the publication of this paper.

\section{Acknowledgments}

The authors are grateful for the financial support from the National Natural Science Foundation of China (51178366), the Technological Project of the Chinese Southern Power Grid Co. Ltd. (K-GD2013-0783), the ESI 1\% Project of WUT (No.43, chenbo), and the Natural Science Foundation of Hubei Province (2014CFA026).

\section{References}

[1] S. W. Doebling, C. R. Farrar, and M. B. Prime, "A summary review of vibration-based damage identification methods," The Shock and Vibration Digest, vol. 30, no. 2, pp. 91-105, 1998.

[2] K. Gurley and A. Kareem, "Applications of wavelet transforms in earthquake, wind and ocean engineering," Engineering Structures, vol. 21, no. 2, pp. 149-167, 1999.

[3] T. Yi, H. Li, and M. Gu, "Full-scale measurements of dynamic response of suspension bridge subjected to environmental loads using GPS technology," Science China Technological Sciences, vol. 53, no. 2, pp. 469-479, 2010.

[4] T.-H. Yi, H.-N. Li, and M. Gu, "Experimental assessment of high-rate GPS receivers for deformation monitoring of bridge," Measurement, vol. 46, no. 1, pp. 420-432, 2013.

[5] A. Masuda, A. Nakaoka, A. Sone, and S. Yamamoto, "Health monitoring system of structures based on orthonormal wavelet transform," Seismic Engineering, vol. 312, no. 1, pp. 161-167, 1995.

[6] A. Sone, S. Yamamoto, A. Masuda, A. Nakaoka, and R. Ashino, "Estimation of cumulative damage of a building with hysteretic restoring force by using wavelet analysis of strong motion records," Japan Structural Construction Engineering, vol. 476, pp. 67-74, 1995.
[7] W. J. Staszewski and G. R. Tomlinson, "Application of the wavelet transform to fault detection in a spur gear," Mechanical Systems and Signal Processing, vol. 8, no. 3, pp. 289-307, 1994.

[8] J. C. Hong, Y. Y. Kim, H. C. Lee, and Y. W. Lee, "Damage detection using the Lipschitz exponent estimated by the wavelet transform: applications to vibration modes of a beam," International Journal of Solids and Structures, vol. 39, no. 7, pp. 18031816, 2002.

[9] J. N. Yang, Y. Lei, and N. E. Huang, "Damage identification of civil engineering structures using Hilbert-Huang transform," in Proceedings of the 3rd International Workshop on Structural Health Monitoring, pp. 544-553, Stanford, Calif, USA, 2001.

[10] Z. Hou and M. Noori, "Application of wavelet analysis for structural health monitoring," in Proceedings of the 2nd International Workshop on Structural Health Monitoring, pp. 946-955, Stanford University, Stanford, Calif, USA, 1999.

[11] Z. Hou, M. Noori, and R. S. Amand, "Wavelet-based approach for structural damage detection," Journal of Engineering Mechanics, vol. 126, no. 7, pp. 677-683, 2000.

[12] H. Sohn, A. N. Robertson, and C. R. Farrar, "Holder exponent analysis for discontinuity detection," Structural Engineering and Mechanics, vol. 17, no. 3-4, pp. 409-428, 2004.

[13] H. T. Vincent, S. L. J. Hu, and Z. Hou, "Damage detection using empirical mode decomposition method and a comparison with wavelet analysis," in Proceedings of the 2nd International Workshop on Structural Health Monitoring, Stanford University, Stanford, Calif, USA, 1999.

[14] J. N. Yang, Y. Lei, S. Lin, and N. Huang, "Hilbert-Huang based approach for structural damage detection," Journal of Engineering Mechanics, vol. 130, no. 1, pp. 85-95, 2004.

[15] Y. L. Xu and J. Chen, "Structural damage detection using empirical mode decomposition: experimental investigation," Journal of Engineering Mechanics, vol. 130, no. 11, pp. 1279-1288, 2004.

[16] B. Chen and Y. L. Xu, "A new damage index for detecting sudden change of structural stiffness," International Journal of Structural Engineering and Mechanics, vol. 26, no. 3, pp. 315-341, 2007.

[17] I. Daubechies, "The wavelet transform, time-frequency localization and signal analysis," IEEE Transactions on Information Theory, vol. 36, no. 5, pp. 961-1005, 1990.

[18] S. Mallat, A Wavelet Tour of Signal Processing, Academic Press, San Diego, Calif, USA, 1998.

[19] B. Chen, Z. W. Chen, G. J. Wang, and W. P. Xie, "Damage detection on sudden stiffness reduction based on discrete wavelet transform," The Scientific World Journal, vol. 2014, Article ID 807620, 16 pages, 2014. 

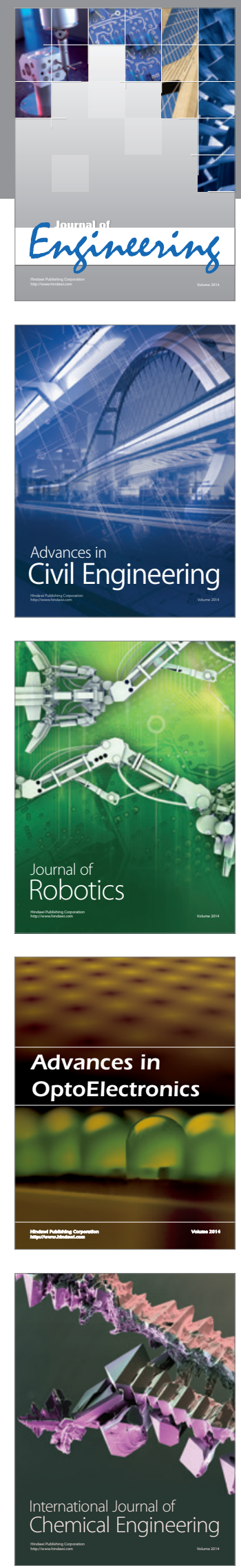

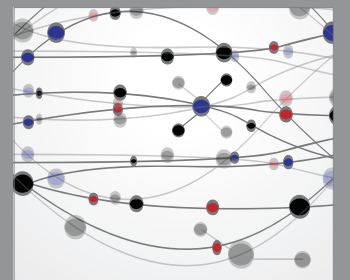

The Scientific World Journal
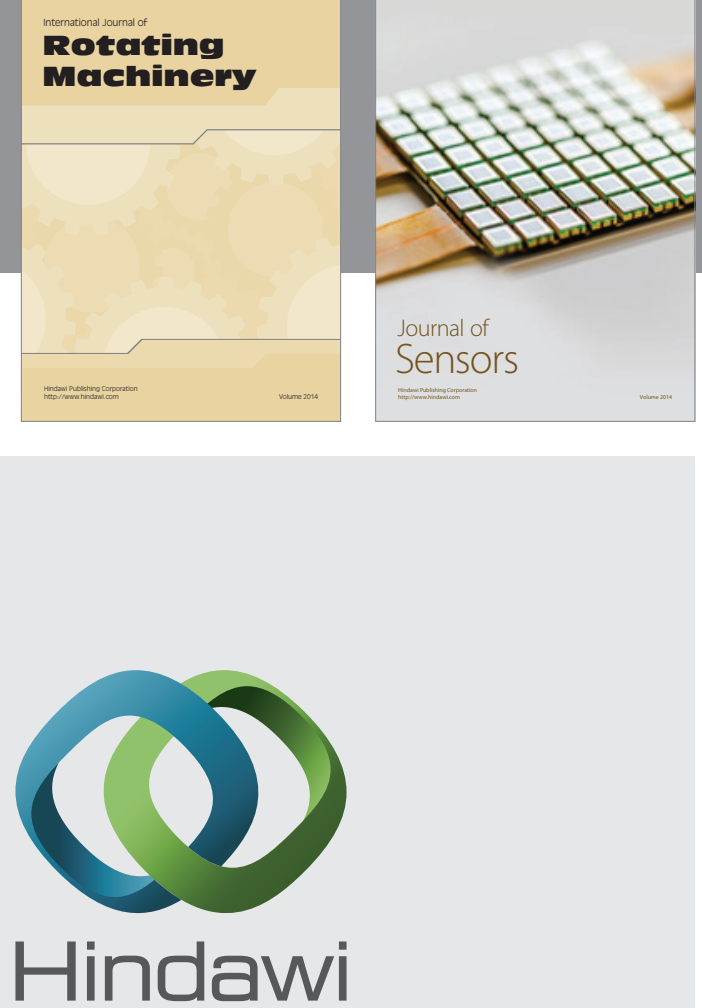

Submit your manuscripts at http://www.hindawi.com
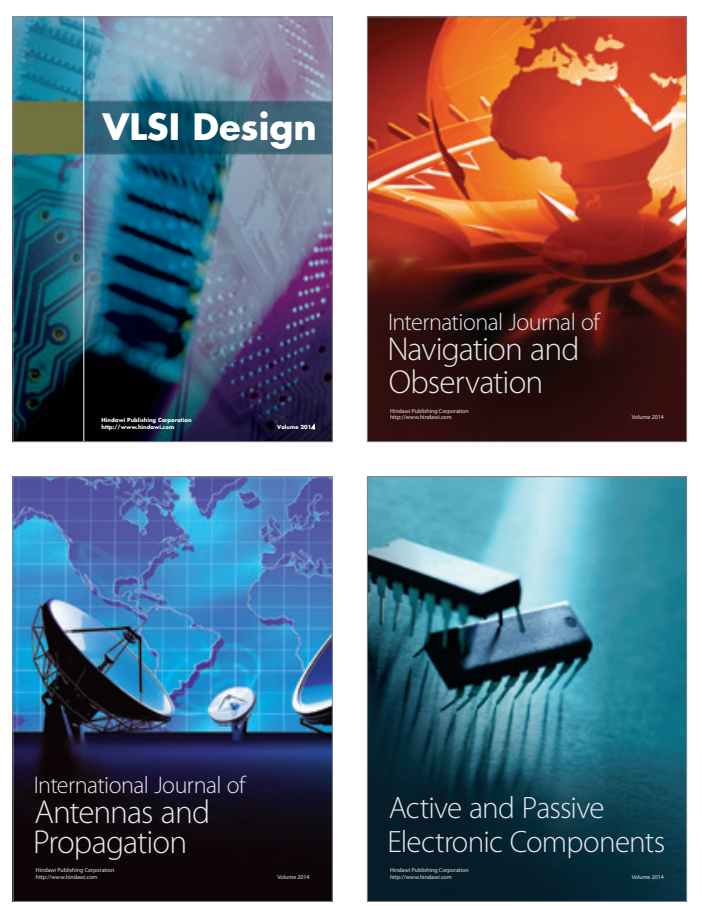
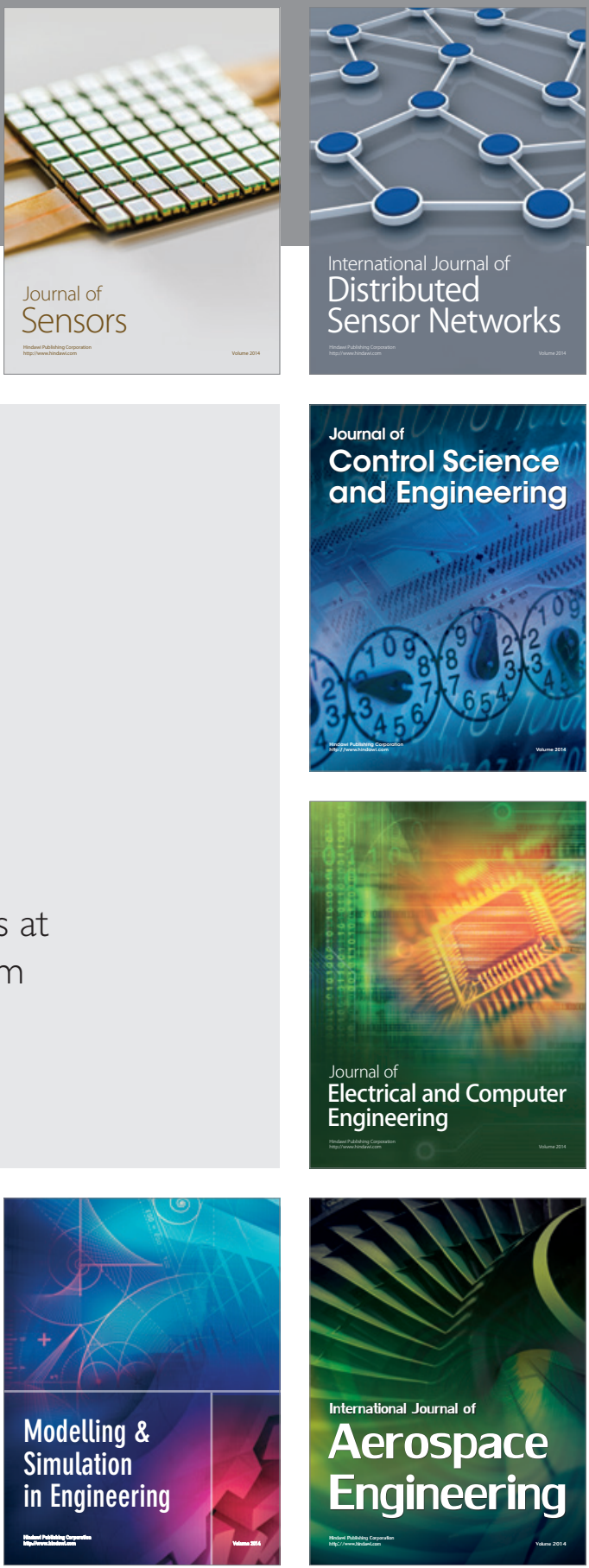

Journal of

Control Science

and Engineering
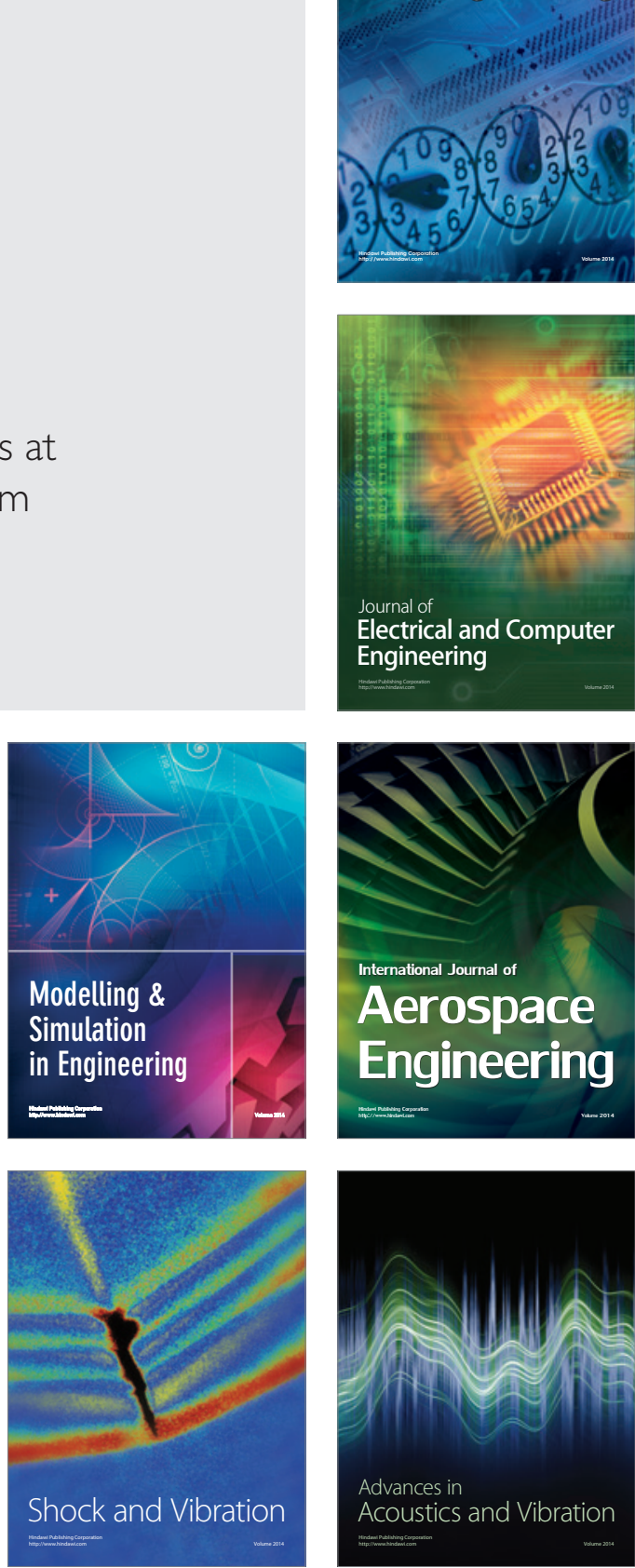